Geochemical data (1ithology, source rock parameters, and hydrocarbons data) of cuttings from the Union 0 il Company OCS Y-0849-1 (Hammerhead No. 1) well

Received 27 February 1989

Geologic Materials Center Data Report No. 116 
February 21, 1989

\author{
Dr. J. Reeder \\ Alaska Division of Geological \\ and Geophysical Surveys \\ P. O. Box 772116 \\ Eagle River, AK 99577
}

Dear John:

Please find attached the geochemical data for Hammerhead-1 cuttings samples. Most of the analytic data are industry standard and a glossary is attached to explain any BP specific terminology. In general, the samples were poor yielding small amounts of cutting material when the mudcake had been washed off. There is also some doubt as to whether drilling additive contamination has occurred but this cannot be conclusively demonstrated. 


\section{GLOSSARY OF TERMS}

\section{Source Rock Parameters}

P1.P2 = Pyrolysis yields obtained from Rock Eval type pyrolysis, equivalent to IFP $\boldsymbol{S}_{1}$ and $\boldsymbol{S}_{2}$.

HI = Hydrogen index derived from

$$
\frac{\mathrm{P} 2}{\mathrm{TOC}} \times 100
$$

units of $\mathrm{mg}$ hydrocarbon/g organic carbon.

GOGI = Gas Oil Generation Index. This is a measurement of source proneness from pyrolysis - GC (pyrograms included) analysis.

$$
\begin{aligned}
& \text { GOGI }=\left(\sum \mathrm{C}_{1}-\mathrm{C}_{5}\right) \\
& \left(\Sigma \mathrm{C}_{6}-\mathrm{C}_{36}\right) \\
& \begin{array}{lll}
\text { Empirically: } & \text { GOGI } \leq 0.2 & =\text { Oil Prone } \\
\text { GOGI } 0.2-0.5 & =\text { Oil }+ \text { Gas Prone } \\
\text { GOGI } \geq 0.5 & =\text { Gas Only prone. }
\end{array}
\end{aligned}
$$

2. Hydrocarbons Data

TSE = Total Soluble Extract, obtained by soxhlet extraction in refluxing dichloromethane.

FRACTIONS:

SAC = Saturate Alkane Content

AROM = Aromatic Hydrocarbon Content

RES = Residue (Equivalent to NSO Content)

All Obtained by HPLC Separation.

Asph = Asphaltene Fraction by precipitation from n-hexane, prior to HPLC separation.

${ }^{13} C_{\text {KER }}=$ Stable carbon isotope composition of kerogen.

${ }^{13} C_{\text {TSE }}=$ Stable carbon isotope composition of Total Soluble Extract. 
BIOMARRER IDENTIPICATION - PENTACYCLIC HYDROCARBONS

BP CODE TENTATIVE ASSIGNMENT BASED ON MASS SPECTROMETRY (m/e 191)

I 9-DODECYLPERHYDROANTHRACENE [INTERNAL STANDARD]

Ts $18 \alpha(\mathrm{H})-22,29,30$-TRISNORNEOHOPANE

$T^{3} \quad 17 \alpha(\mathrm{H})-22,29,30$-TRISNORHOPANE

0 17 $\beta(\mathrm{H})-22,29,30$-TRISNORHOPANE

$17 \alpha(\mathrm{H})-25,30$-BISNOBHOPANE

$X \quad 17 \alpha(B), 18 \alpha(B), 21 \beta(B)-28,30$-BISNORHOPANE

$Y \quad 17 \alpha(\mathrm{B})-25$-NORBOPANE

D $17 \alpha($ B $), 21 \beta($ B $)-30$-NORHOPANE

$\pi \quad \mathrm{C}_{30}$ PENTACYCLIC TRITERPANE

A $17 \beta(\mathrm{B}), 21 \alpha(\mathrm{B})$-30-NORMORETANE

B $18 \alpha(\mathrm{H})$-OLEANANE

G $17 \alpha(\mathrm{H}), 21 \beta(\mathrm{H})-\mathrm{HOPANE}$

B $17 \beta(\mathrm{H}), 21 \beta(\mathrm{H})-30$-NORHOPANE

$\mathrm{K} \quad 17 \beta(\mathrm{H}), 21 \alpha(\mathrm{B})$-MORETANE

$\mathrm{N} \quad(22 \mathrm{~S})-17 \alpha(\mathrm{B}), 21 \beta(\mathrm{H})-30$-METHYLHOPANE

$0 \quad(22 R)-17 \alpha(B), 21 \beta(\mathrm{H})-30$-METHYLBOPANE

S GAMMACERANE

P $17 B(\mathrm{H}), 21 \beta(\mathrm{H})-\mathrm{HOPANE}$

$R \quad 17 \beta(\mathrm{B}), 21 \alpha(\mathrm{H})-30$-METHYLMORETANE

U (22S) $-17 \alpha(\mathrm{H}), 21 \beta(\mathrm{B})-30$-ETHYLHOPANE

$V \quad(22 R)-17 \alpha(B), 21 \beta(B)-30$-ETHYLHOPANE

$\mathrm{J} \quad 17 \beta(\mathrm{B}), 21 \beta(\mathrm{B})$-METHYLHOPANE

$\alpha \quad$ (22S) $-17 \alpha(\mathrm{B}), 21 \beta(\mathrm{B})-30-n-\mathrm{PROPYLHOPANE}$

$\beta \quad(22 R)-17 \alpha(B), 21 \beta(B)-30-n$-PROPYLHOPANE

L $\quad 17 \beta(B), 21 \beta(B)$-ETHYLHOPANE

$\gamma \quad$ (22S) $-17 \alpha(\mathrm{B}), 21 \beta(\mathrm{B})-30-n-B U T Y L H O P A N E$

$\delta$ (22R) $-17 \alpha(B), 21 \beta(B)-30-n$-BUTYLHOPANE

$\varepsilon \quad$ (22S) $-17 \alpha(B), 21 \beta(B)-30-n-P E N T Y L H O P A N E$

(22R) $-17 \alpha(\mathrm{B}), 21 \beta(\mathrm{B})-30-\mathrm{n}-\mathrm{PENTYLHOPANE}$

BIOMARKER IDENTIFICATION - STERANES

BP CODE TENTATIVE ASSIGNMENT BASED ON MASS SPECTROMETRY (m/e 217)

\begin{tabular}{|c|c|}
\hline $\begin{array}{l}10 \\
11 \\
13 \\
14 \\
15 \\
16 \\
18 \\
19 \\
20 A \\
20 B \\
21 A \\
21 B \\
22 \\
25 \\
27 \\
29 \\
33 \text { A } \\
33 B \\
34 \\
36 \\
39 \\
40 \\
41\end{array}$ & 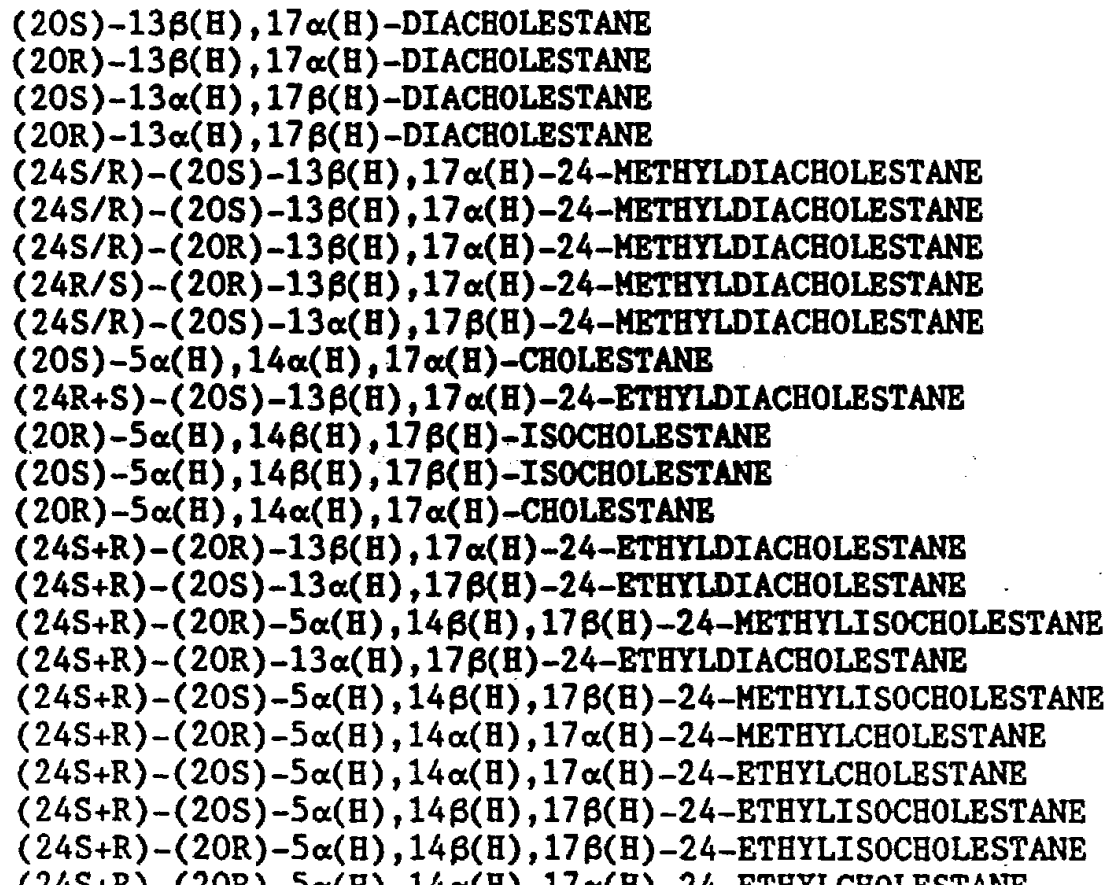 \\
\hline
\end{tabular}


BP CODE TENTATIVE ASSIGNMENT BASED ON MASS SPECTROMETRY (m/e 253 mass fragmentogram)

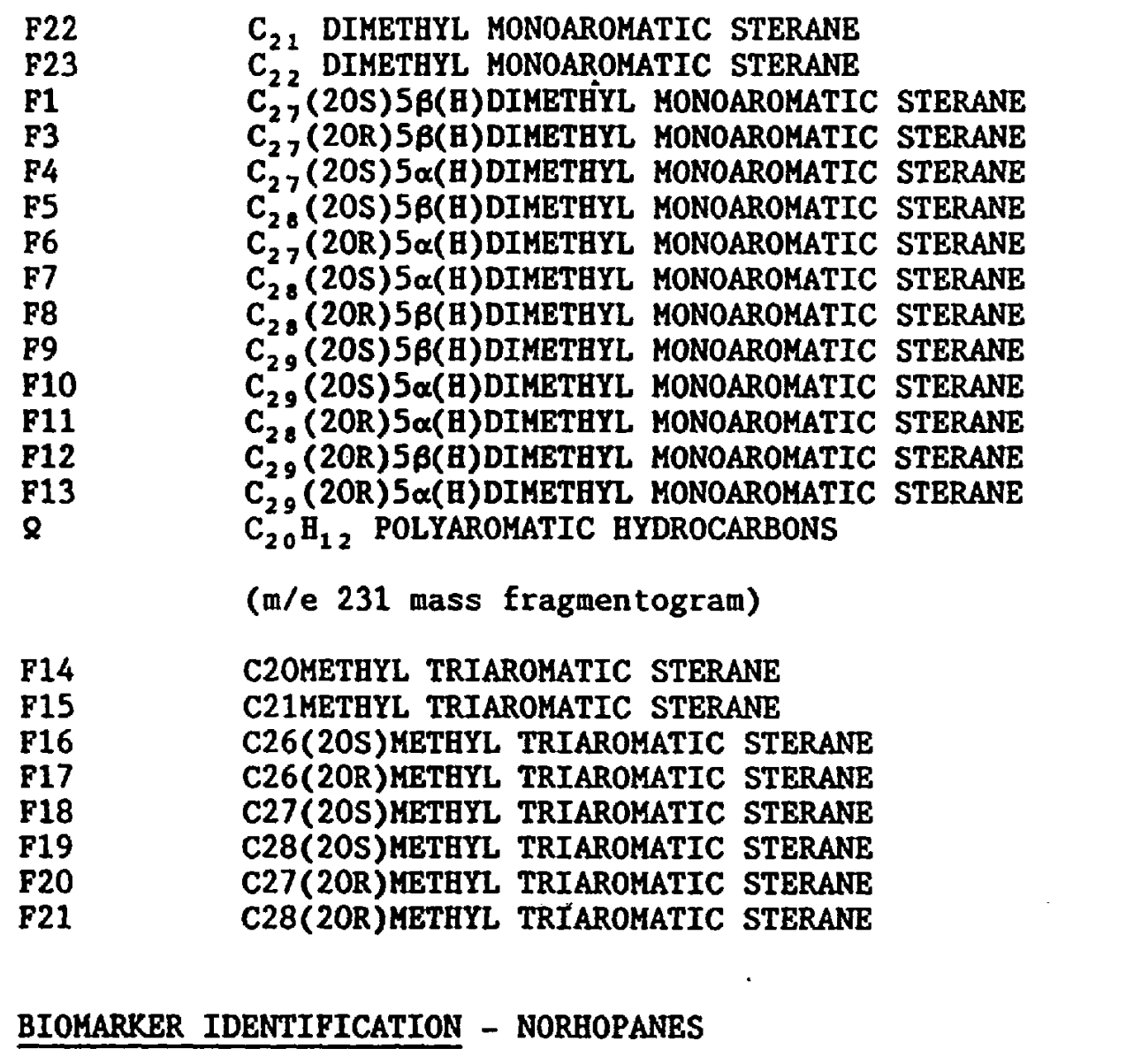

BP CODE TENTATIVE ASSIGNMENT BASED ON MASS SPECTROMETRY (m/e 177)

\begin{tabular}{|c|c|}
\hline $\begin{array}{l}\text { V } \\
Y \\
D \\
\text { C1 } \\
\text { G } \\
\text { C2 } \\
\text { C3 } \\
\text { C4 } \\
\text { C5 } \\
\text { C6 } \\
\text { C7 } \\
\text { C8 } \\
\text { C9 } \\
\text { C10 }\end{array}$ & 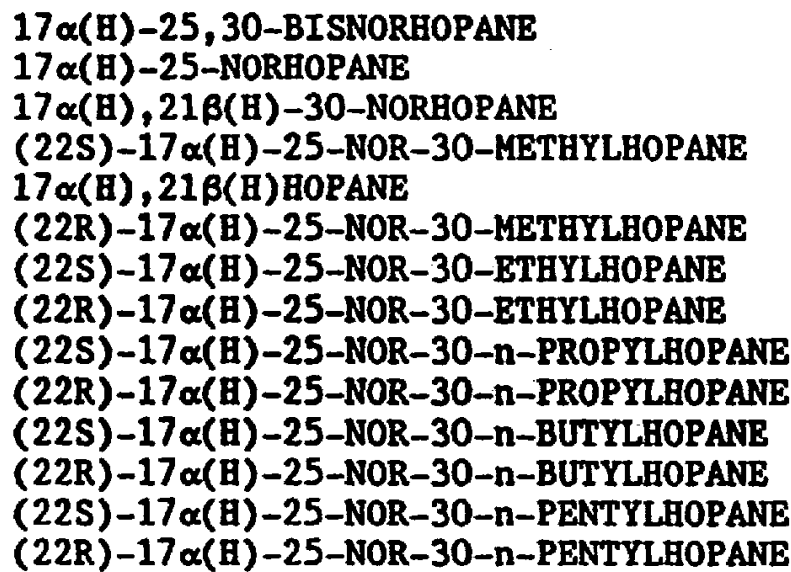 \\
\hline
\end{tabular}




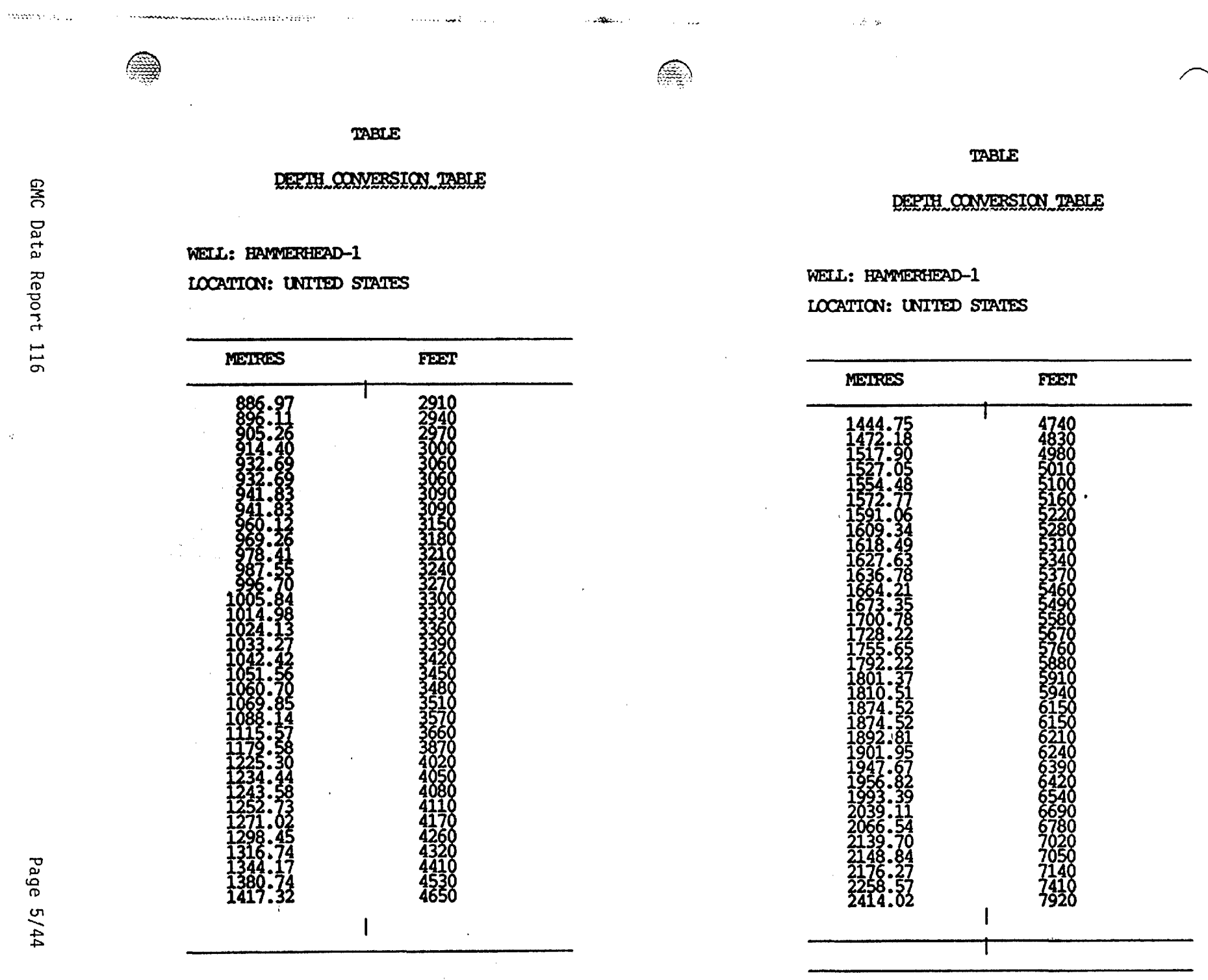


TABLE

5

LITHOLOGY AND STRATIGRAPHY

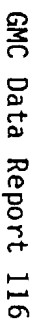

WELL: HAMEREIEAD-1

LOCATION: UNITED STATES

\begin{tabular}{|c|c|c|c|c|}
\hline$\frac{\text { DEPTH }}{(\mathrm{m})}$ & DEPIH RANEE & FORMATION & ACE & LITHOLOGY \\
\hline $\begin{array}{l}886.97 \\
896 \cdot \frac{11}{2} \\
905 \cdot 26 \\
914.40\end{array}$ & $\begin{array}{l}877.82-886.97 \\
886.97-896 \cdot \frac{11}{26} \\
896 \cdot \frac{1}{1}-905.26 \\
905.26-914.40\end{array}$ & \multirow{10}{*}{ T.2.0. } & $\begin{array}{l}\text { TERTIAAY } \\
\text { TERTIARY } \\
\text { TERTIARY } \\
\text { TERTIARY }\end{array}$ & \multirow{11}{*}{ 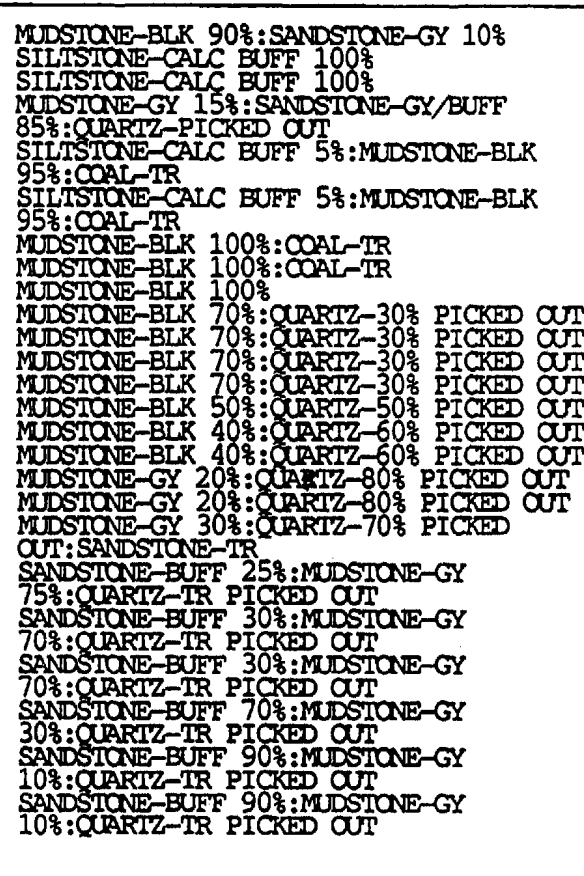 } \\
\hline 932.69 & $923.54-932.69$ & & TERTIARY & \\
\hline 932.69 & $923.54-932.69$ & & TERTIAARY & \\
\hline $\begin{array}{r}941.83 \\
941: 83 \\
960.12 \\
969.26 \\
978.41 \\
987.55 \\
996.70 \\
1005.84 \\
1014.98 \\
1024.13 \\
1033.27 \\
1042.42 \\
1051.56\end{array}$ & $\begin{array}{r}932.69-941.83 \\
932.69-941.83 \\
950.98-960.12 \\
960.12-969.26 \\
969.26-978.41 \\
978.41-987.55 \\
987.55-996.70 \\
996.70-1005.84 \\
1005.84-1014.98 \\
1014.98-1024.13 \\
1024.13-1033.27 \\
1033.27-1042.42 \\
1042.42-1051.56\end{array}$ & & 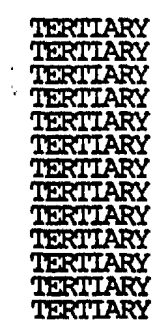 & \\
\hline 1060.70 & $1051.56-1060.70$ & & TERTIARY & \\
\hline 1069.85 & $1060.70-1069.85$ & & TERTIARY & \\
\hline 1088.14 & $1078.99-1088.14$ & & TERTIAARY & \\
\hline 1115.57 & $1106.42-1115.57$ & & TERTIARY & \\
\hline 1179.58 & $1170.43-1179.58$ & & TERITIARY & \\
\hline 1225.30 & $1216.15-1225.30$ & & TERTIIARY & \\
\hline & | & | & 1 & \\
\hline
\end{tabular}


TABLE

LITHOLOGY AND STRATIGRAPHY

WEIL: HAMMERHEAD-1

LOCATION: UNITED STATES

\begin{tabular}{|c|c|c|c|c|}
\hline$\frac{\text { DEPTH }}{(\mathrm{m})}$ & DEPTH RANGE & FORMATION & $A G E$ & LITHOLOGY \\
\hline 1234.44 & $1225.30-1234.44$ & & TERIIARY & \multirow{19}{*}{ 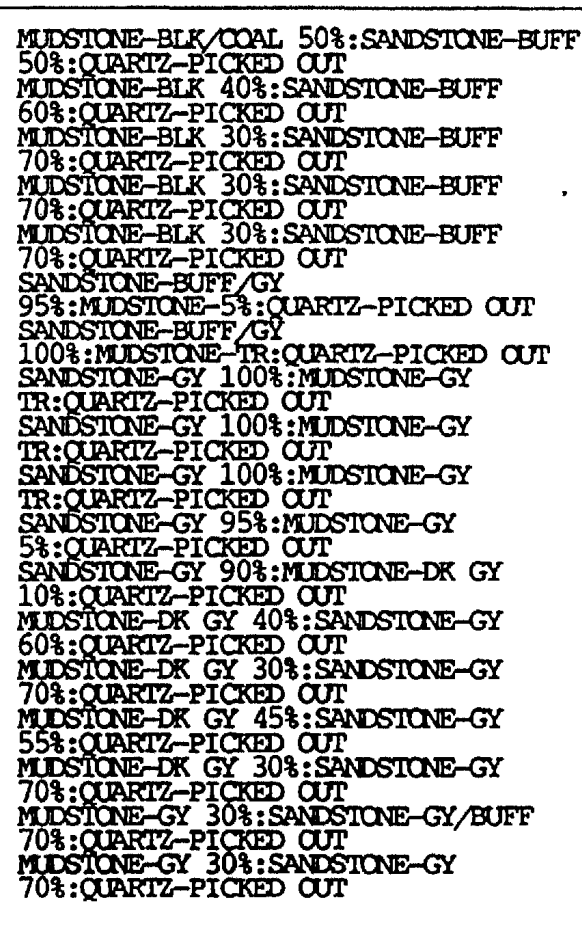 } \\
\hline 1243.58 & $1234.44-1243.58$ & & TERTIIARY & \\
\hline 1252.73 & $1243.58-1252.73$ & & TERTIARY & \\
\hline 1271.02 & $1261.87-1271.02$ & & TERIIIARY & \\
\hline 1298.45 & $1289.30-1298.45$ & & TERTIARY & \\
\hline 1316.74 & $1307.59-1316.74$ & & TERTIARY & \\
\hline 1344.17 & $1335.02-1344.17$ & & TERTIARY & \\
\hline 1380.74 & $1371.60-1380.74$ & & TERTIARY & \\
\hline 1417.32 & $1408.18-1417.32$ & & TERTIARY & \\
\hline 1444.75 & $1435.61-1444.75$ & & TERTIIARY & \\
\hline 1472.18 & $1463.04-1472.18$ & & TERTIARY & \\
\hline 1517.90 & $1508.76-1517.90$ & & TERTIARY & \\
\hline 1527.05 & $1517.90-1527.05$ & & TERTIARY & \\
\hline 1554.48 & $1545.34-1554.48$ & & TERTIIARY & \\
\hline 1572.77 & $1563.62-1572.77$ & & TERIIAARY & \\
\hline 1591.06 & $1581.91-1591.06$ & & TERIIAFY & \\
\hline 1609.34 & $1600.20-1609.34$ & & TERIIAARY & \\
\hline 1618.49 & $1609.34-1618.49$ & & TERTIARY & \\
\hline & 1 & & & \\
\hline
\end{tabular}

MUDSTONE-BLK COAL 508: SANDSTONE-BUFF

50\%: QUARTZ-PICKED O

MUDSIONE-BLK 40\%: SANDSTONE-BUFE

$70 \%:$ OARTR-PICKED QS

SANDSTONE-BUFF 5.. 100\%:MUDSTONE-TR-OAARTZ-PICKED OT

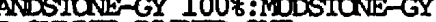
SART

SANDSTIONE-GY 1008 :MLDSTONE-GY SANDSTONE-GY 100\%:MDSTONE-GY IR:OAARIZ-PICKED OUT

TIR GY 40\%:SANDSTONE-GY :QARTZ-PICKMD OUT

SOSDNE-DK GY 308:SANDSTONE-GY 70\%:OUARIZ-PICKED OUI

促 
TABLE

LITHOLOGY AND STRATIGRAPHY

WELL: HAMMERHEAD-1

LOCATION: UNTTED SIATES

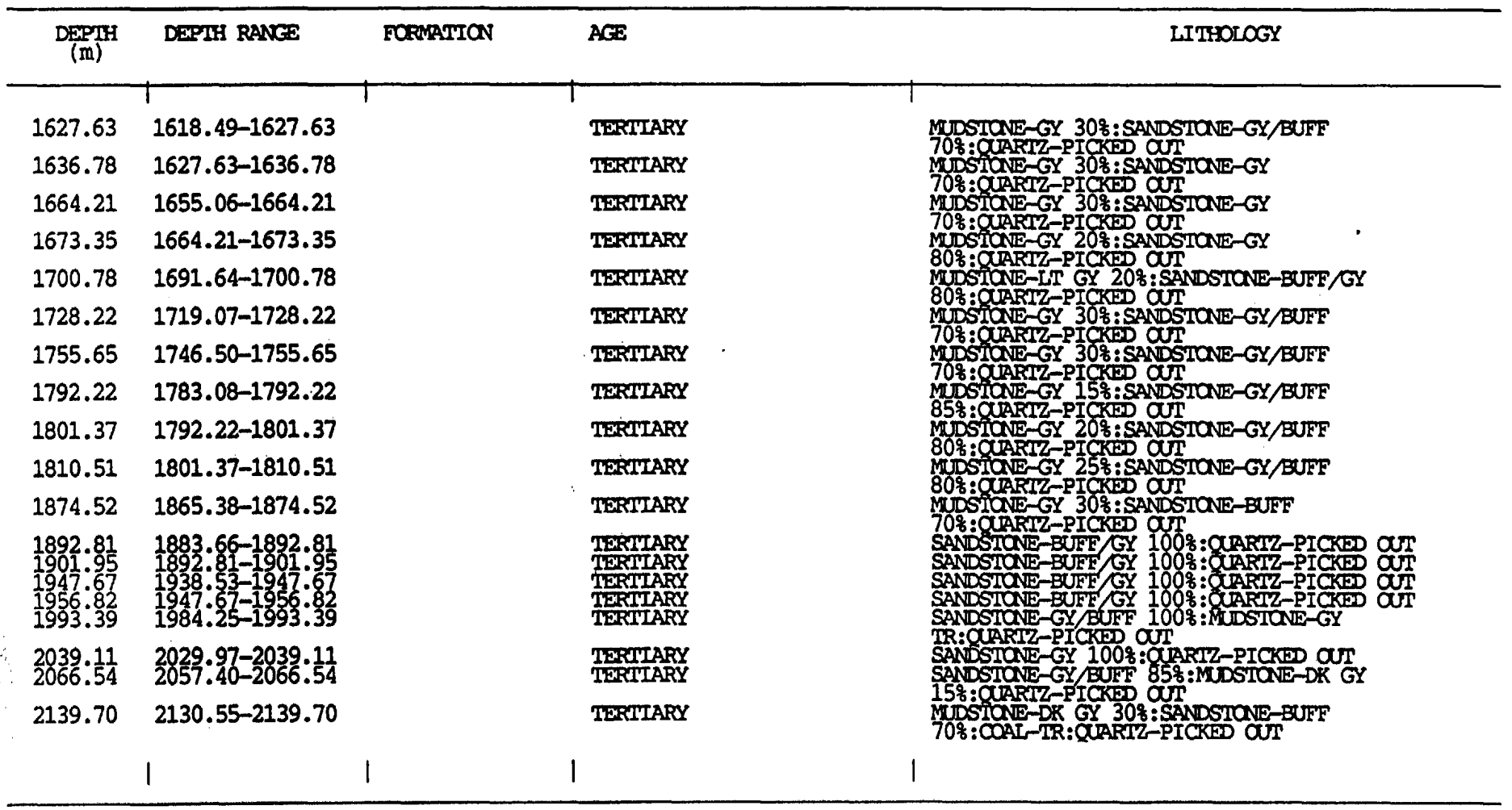




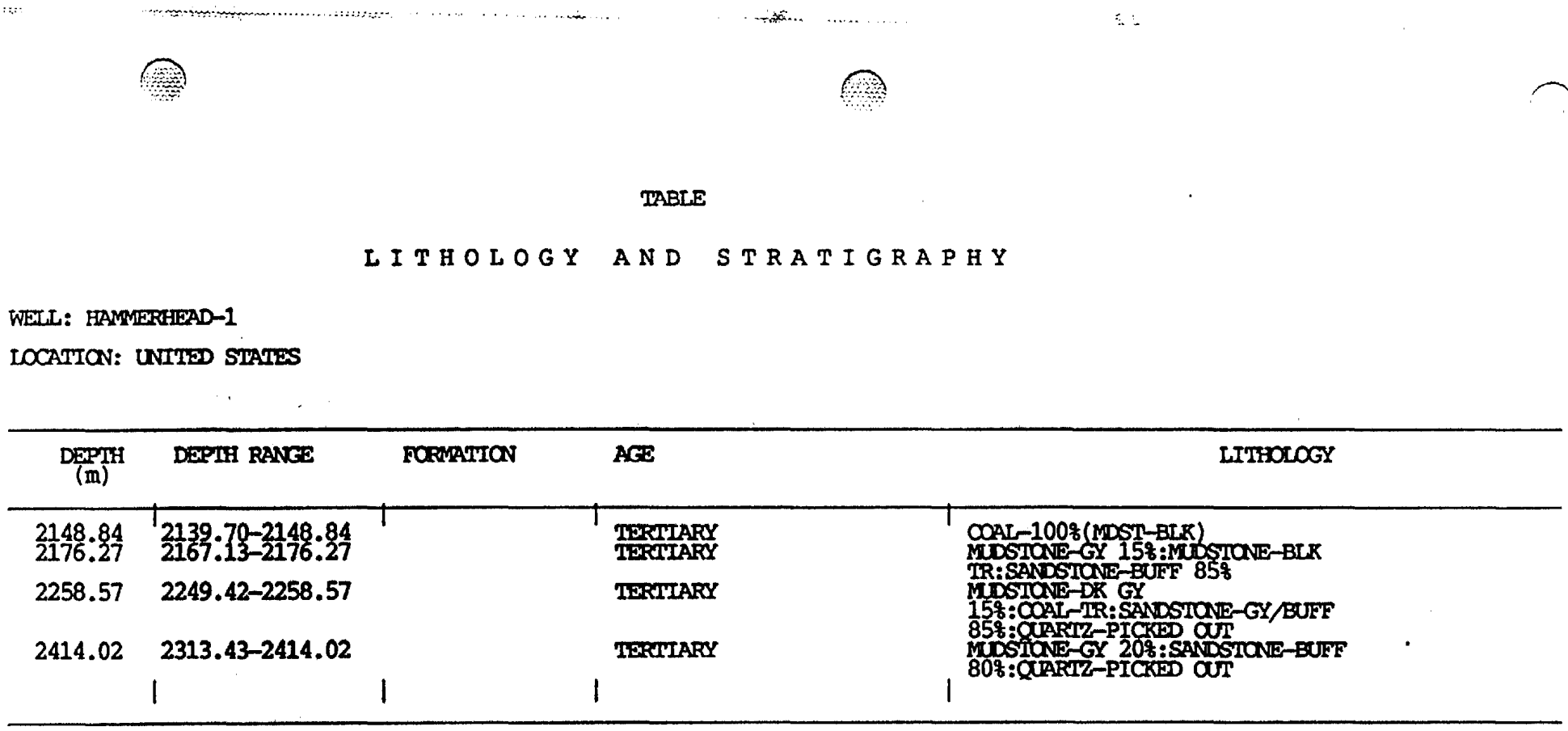

WETC: HAMMERHEAD-1

LOCATION: UNTTED STATES 


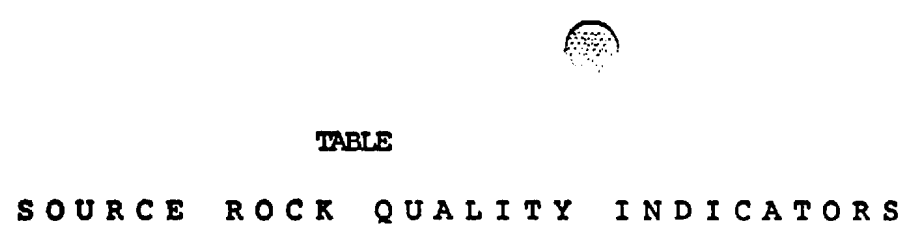

NETL: FAMMERETPAD-1

LOCATION: UNTHED STHMTES

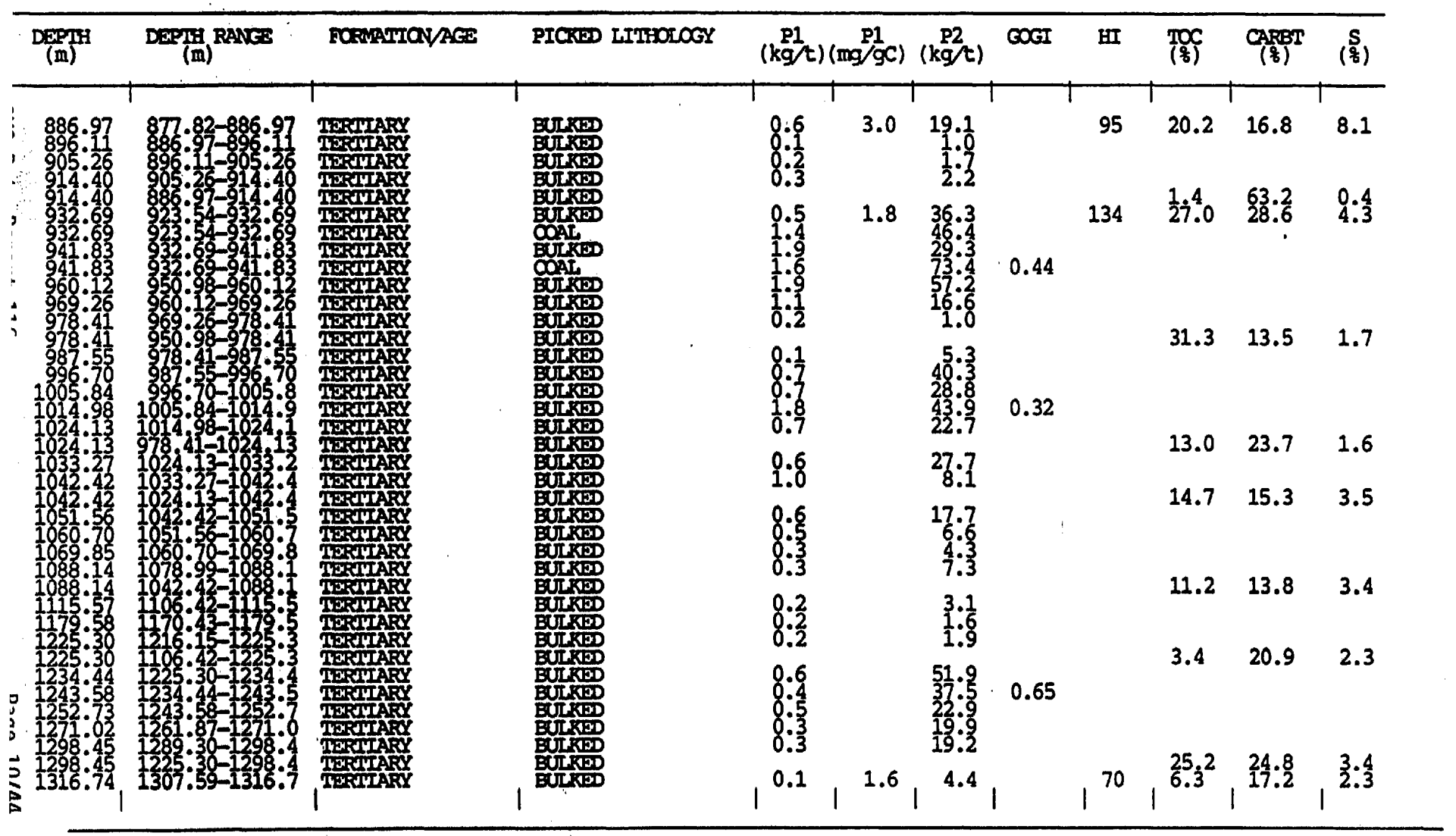


TABLE

SOURCE ROCR QUALITY INDICATORS

WELL: HAMMERTEEAD-1

LOCATION: UNTHED STATES

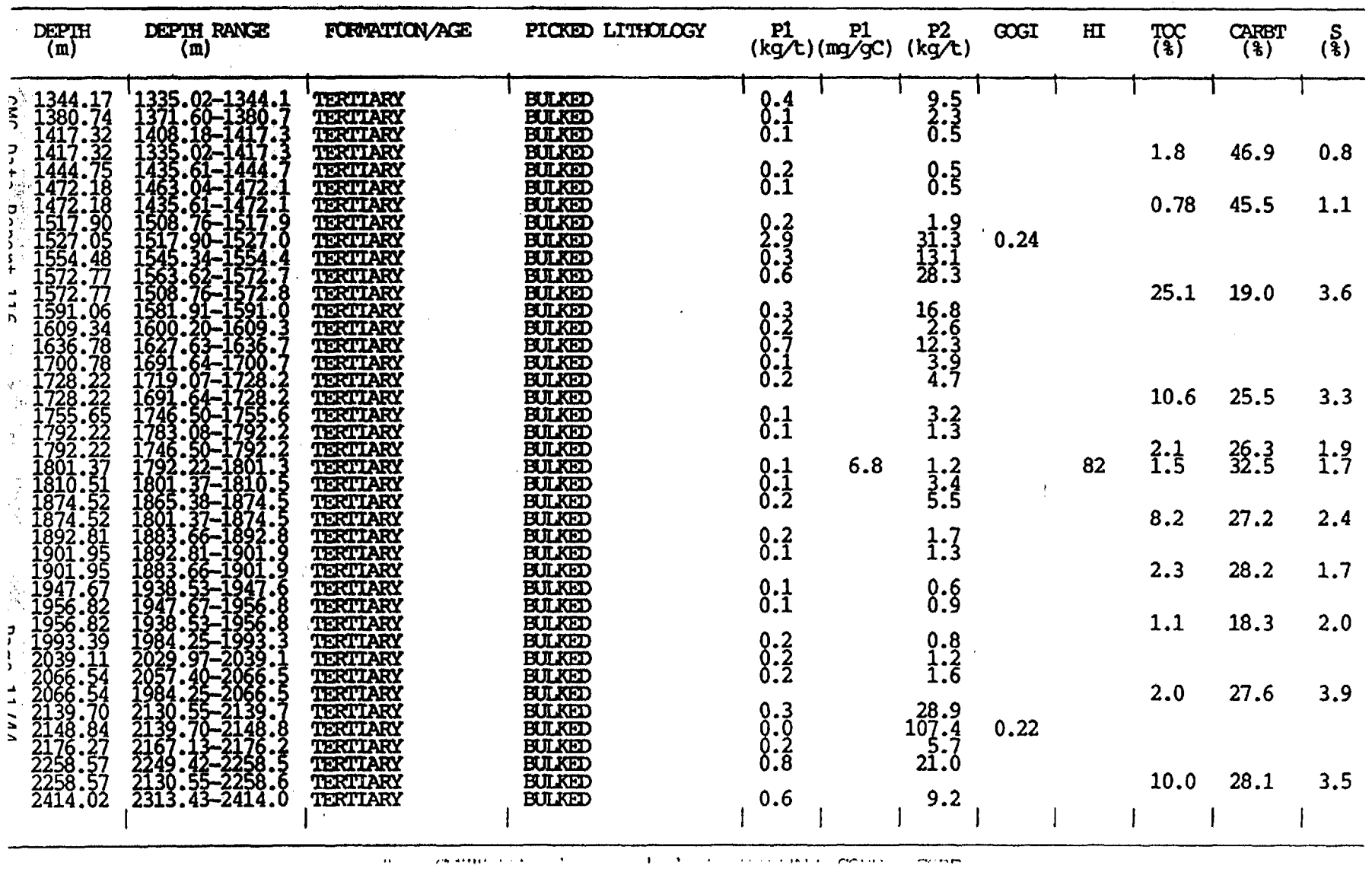


TABLE

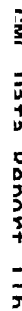

PYROLYSIS-GC DATA

WELL: HAMRERTEAD-1

LOCATION: UNITED STATES

\begin{tabular}{|c|c|c|c|c|c|c|c|c|c|}
\hline$\frac{D \text { DPTH }}{(\mathrm{m})}$ & FCRMATION/AGE & $\frac{C 1-c 5}{(8)}$ & $\frac{C 6-c 9}{(8)}$ & $\mathrm{ClO}_{(8)}$ & $\mathrm{Cl}_{(\mathrm{g})}$ & $\frac{C^{23-C 36}}{(8)}$ & GOGI & HII & ${ }_{(8)}$ \\
\hline $\begin{array}{r}941.83 \\
1014.98 \\
1243.58 \\
1527.05 \\
2148.84\end{array}$ & 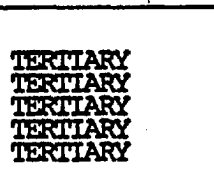 & \begin{tabular}{|l} 
\\
30 \\
24 \\
39 \\
19 \\
18 \\
18
\end{tabular} & $\begin{array}{l}\frac{11}{11} \\
\frac{11}{11} \\
\frac{11}{14}\end{array}$ & $\begin{array}{ll} & 17 \\
21 \\
23 \\
14 \\
13 \\
\end{array}$ & $\begin{array}{l}23 \\
23 \\
16 \\
28 \\
25\end{array}$ & $\begin{array}{l}18 \\
19 \\
25 \\
27\end{array}$ & $\begin{array}{l}0.44 \\
8.32 \\
8.65 \\
8.24 \\
8.22\end{array}$ & T & 1 \\
\hline
\end{tabular}




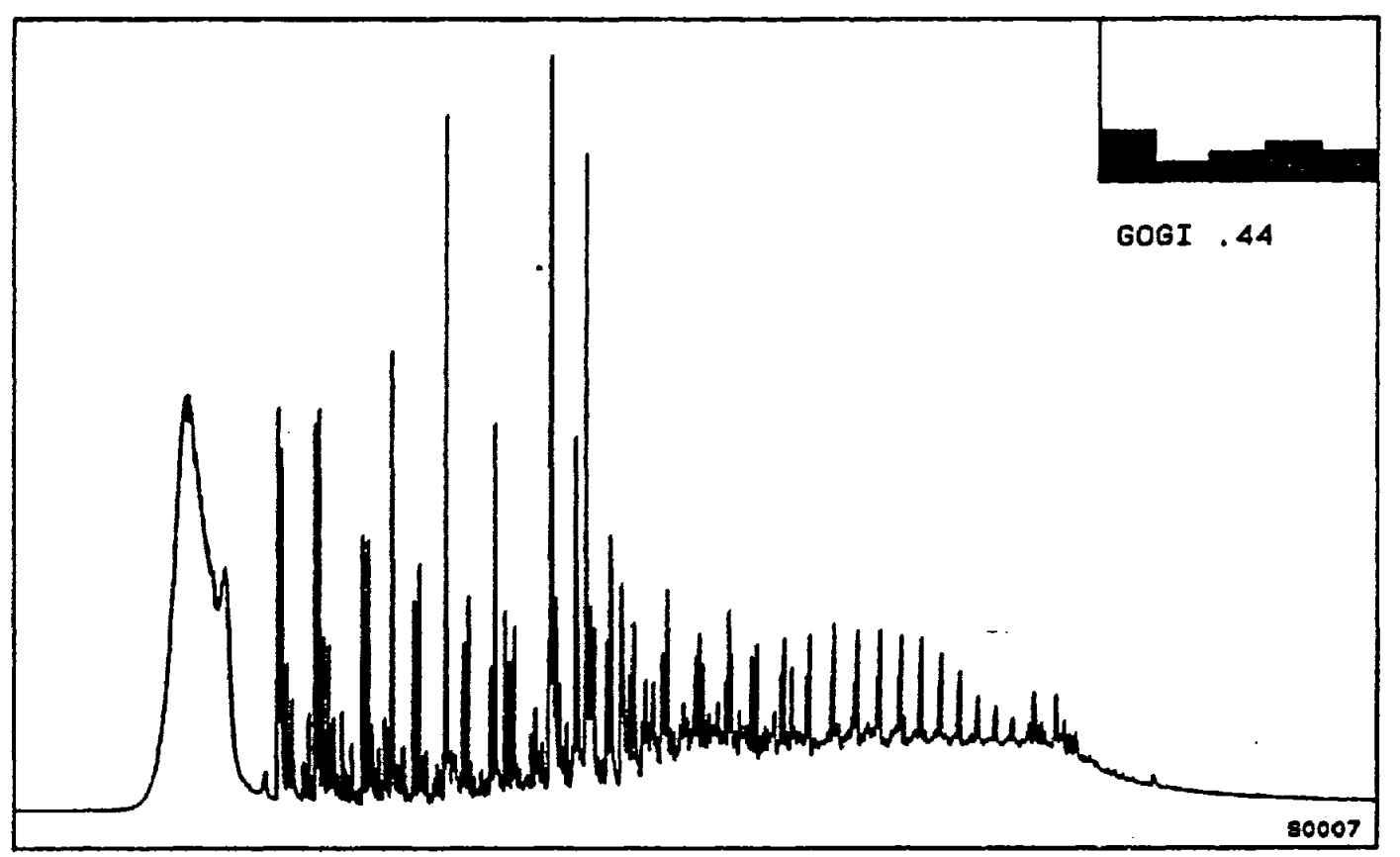

$941.83 \mathrm{~m}$ (CUT)

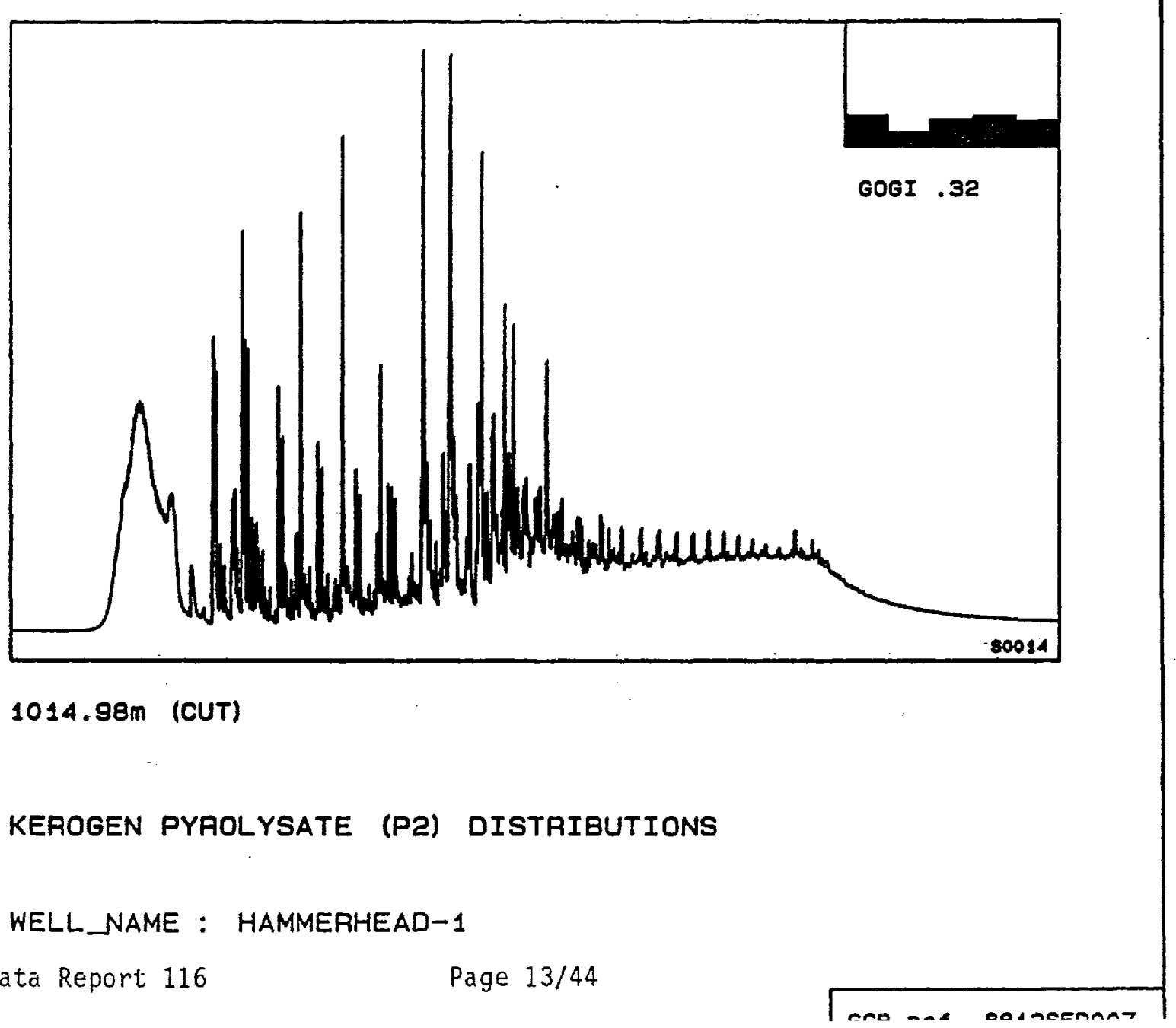




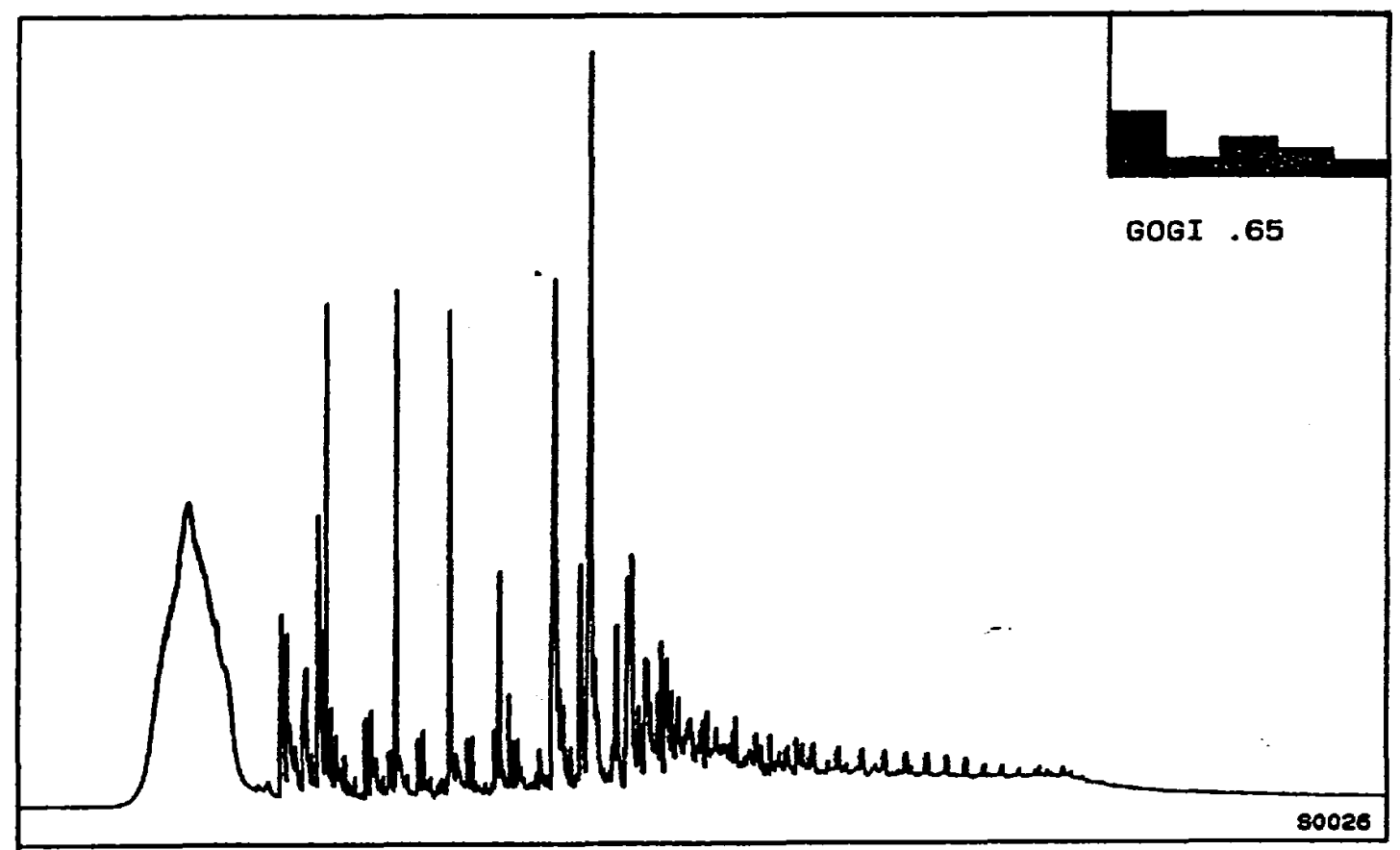

1243.58m (CUT)

(.)

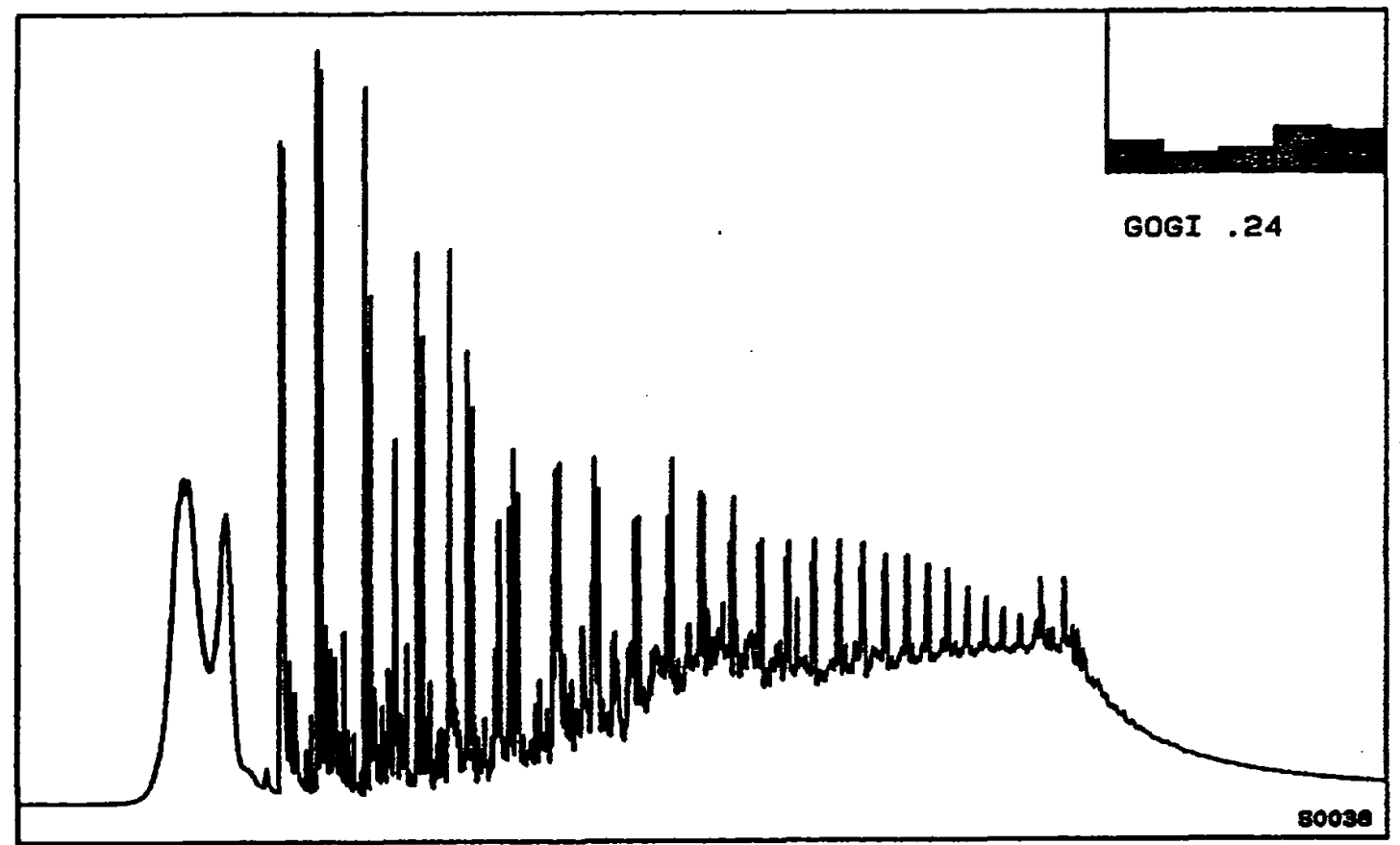

$1527.05 \mathrm{~m}$ (CUT)

KEROGEN PYROLYSATE (PZ) DISTAIBUTIONS

WELL NAME : HAMMERHEAD-1

GMC Data Report 116

Page $14 / 44$

GCR ref. B812SFDOOT 


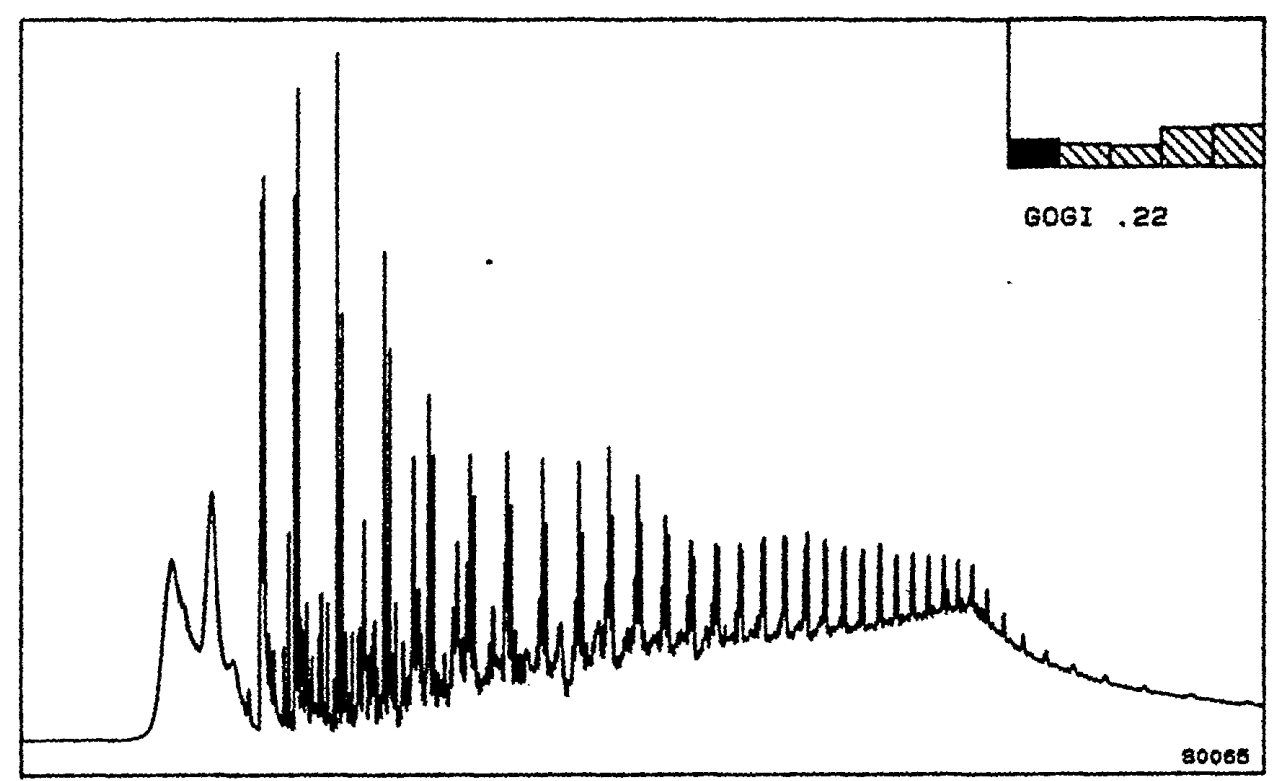

$2148.84 \mathrm{~m}$ (CUT)

KEROGEN PYAOLYSATE (PZ) DISTRIBUTIONS

WELL NAME : HAMMERHEAD-1

GMC Data Report 116

Page $15 / 44$

GCB ref. B812SED007 
.

TABLE

SOLUBLE EXTRACT DATA-1

WELL: FAMPERHEAD-1

LOCATION: UNITED STATES

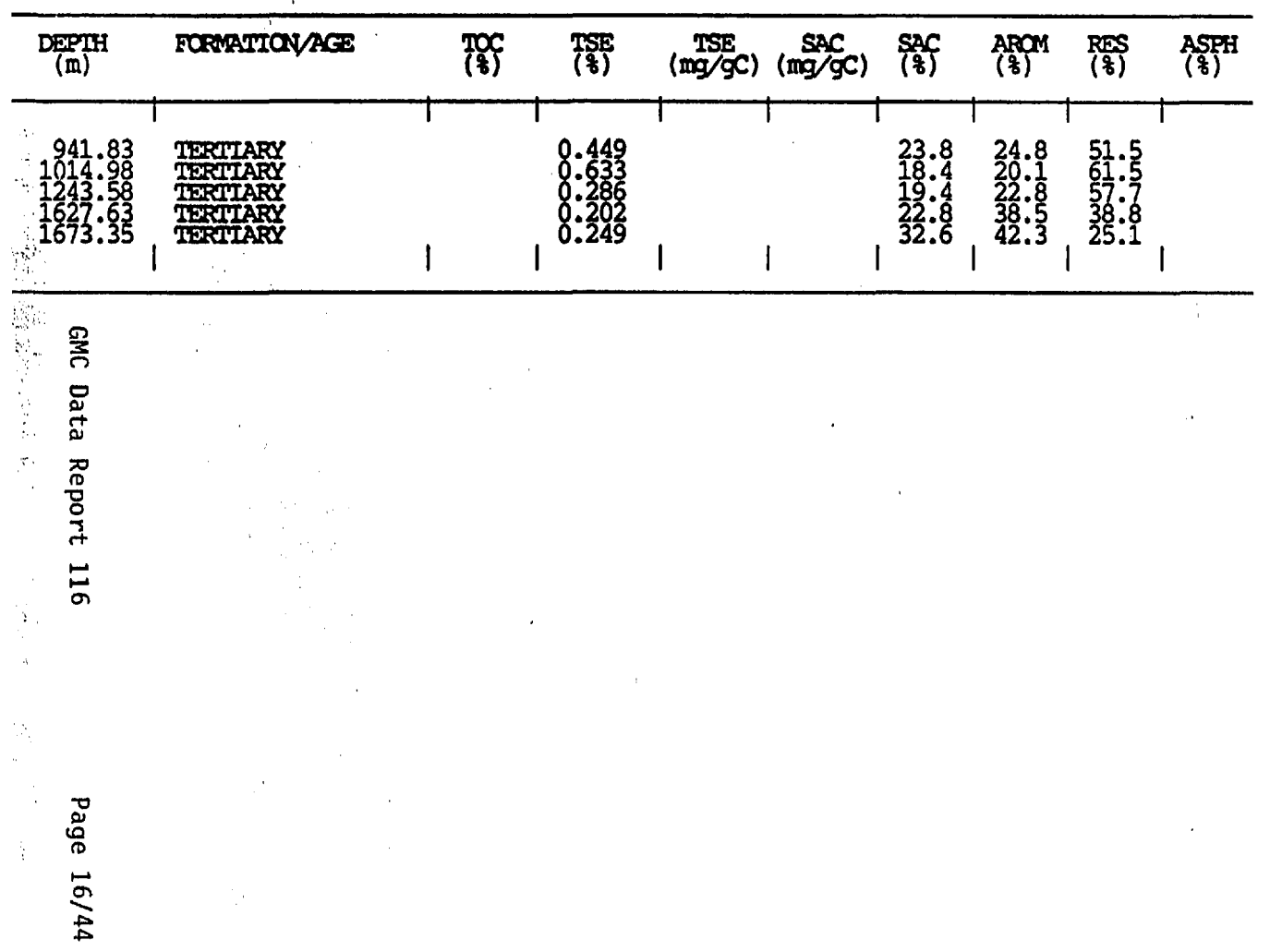




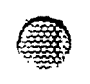

TABLE

CARBON ISOTOPE RATIOS

WETL: HAMMEREFAD-1

LOCATTON: UNITED STATES

\begin{tabular}{|c|c|c|c|}
\hline$\frac{\text { DEPTH }}{(\mathrm{m})}$ & $\begin{array}{l}\text { PICKSD } \\
\text { LITHOCY }\end{array}$ & SAMPLE & $\begin{array}{l}\text { ISOTOPE RATTO } \\
\text { (per mil) }\end{array}$ \\
\hline $\begin{array}{r}941.83 \\
941.83 \\
1014.98 \\
1014.98 \\
1243.58 \\
1243.58\end{array}$ & 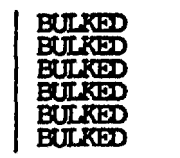 & $\begin{array}{l}\text { TSE } \\
\text { KERR } \\
\text { TSSE } \\
\text { KISR } \\
\text { TSEE } \\
\text { KERR }\end{array}$ & $\begin{array}{l}-28.8 \\
-25: 0 \\
-27.6 \\
-25: 0 \\
-28.4 \\
-25.2\end{array}$ \\
\hline
\end{tabular}

C-12 C-13 ISOTOPIC RATMOS ARE RETATTVE TO

疍

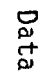

总

ᄀ

के

$D$
$\stackrel{0}{0}$
$\stackrel{D}{D}$
$\stackrel{D}{D}$ 


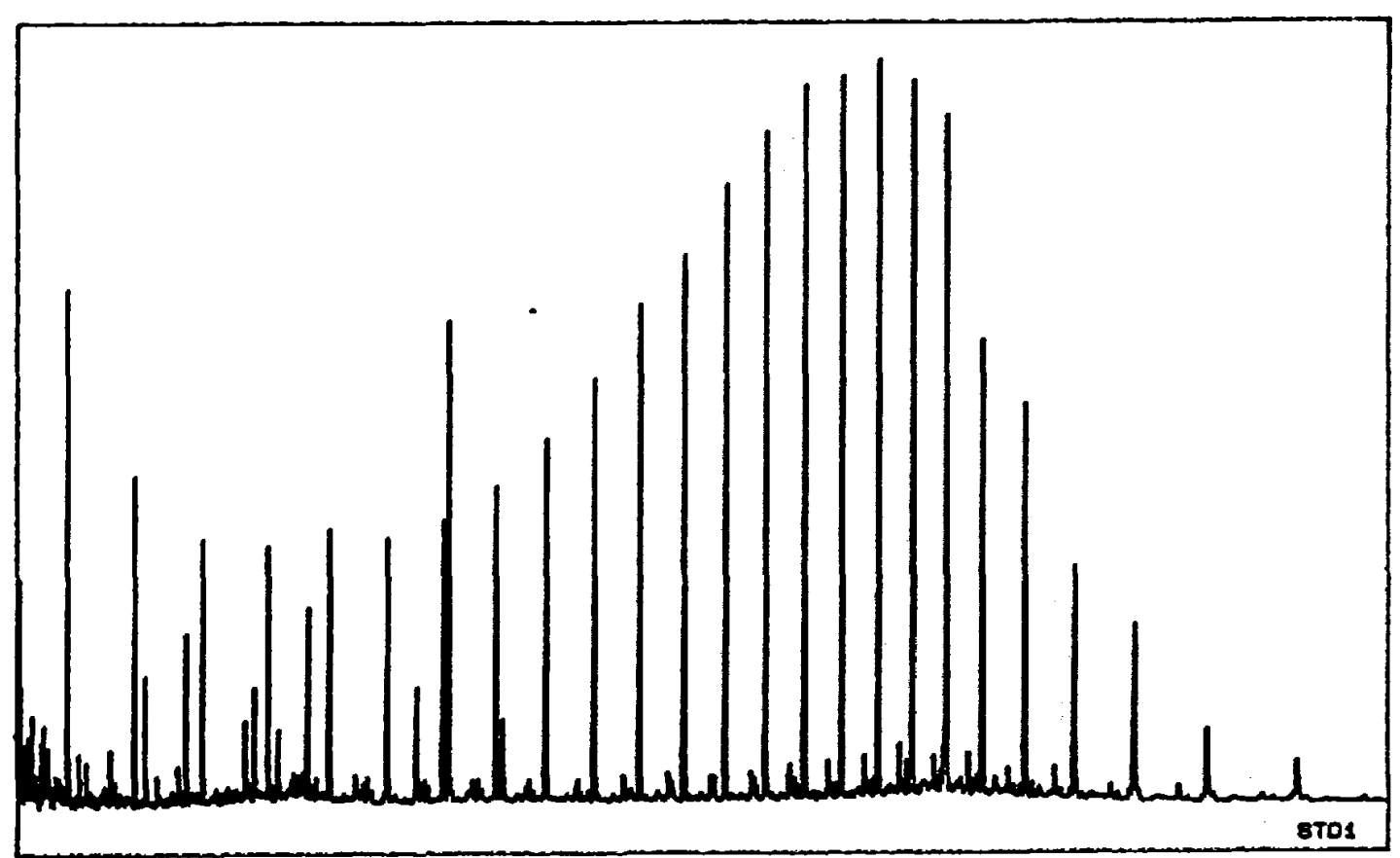

STANDARD

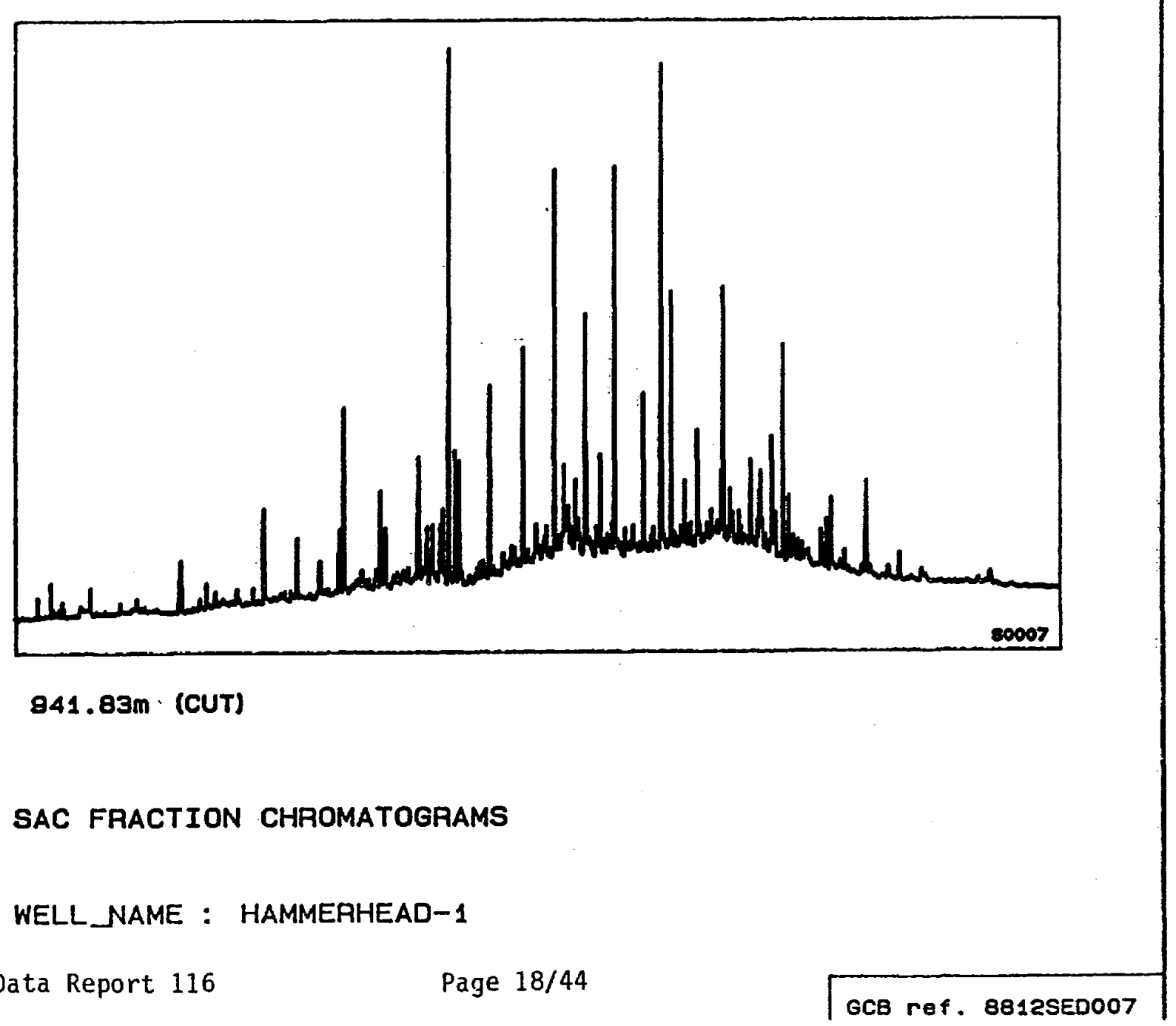




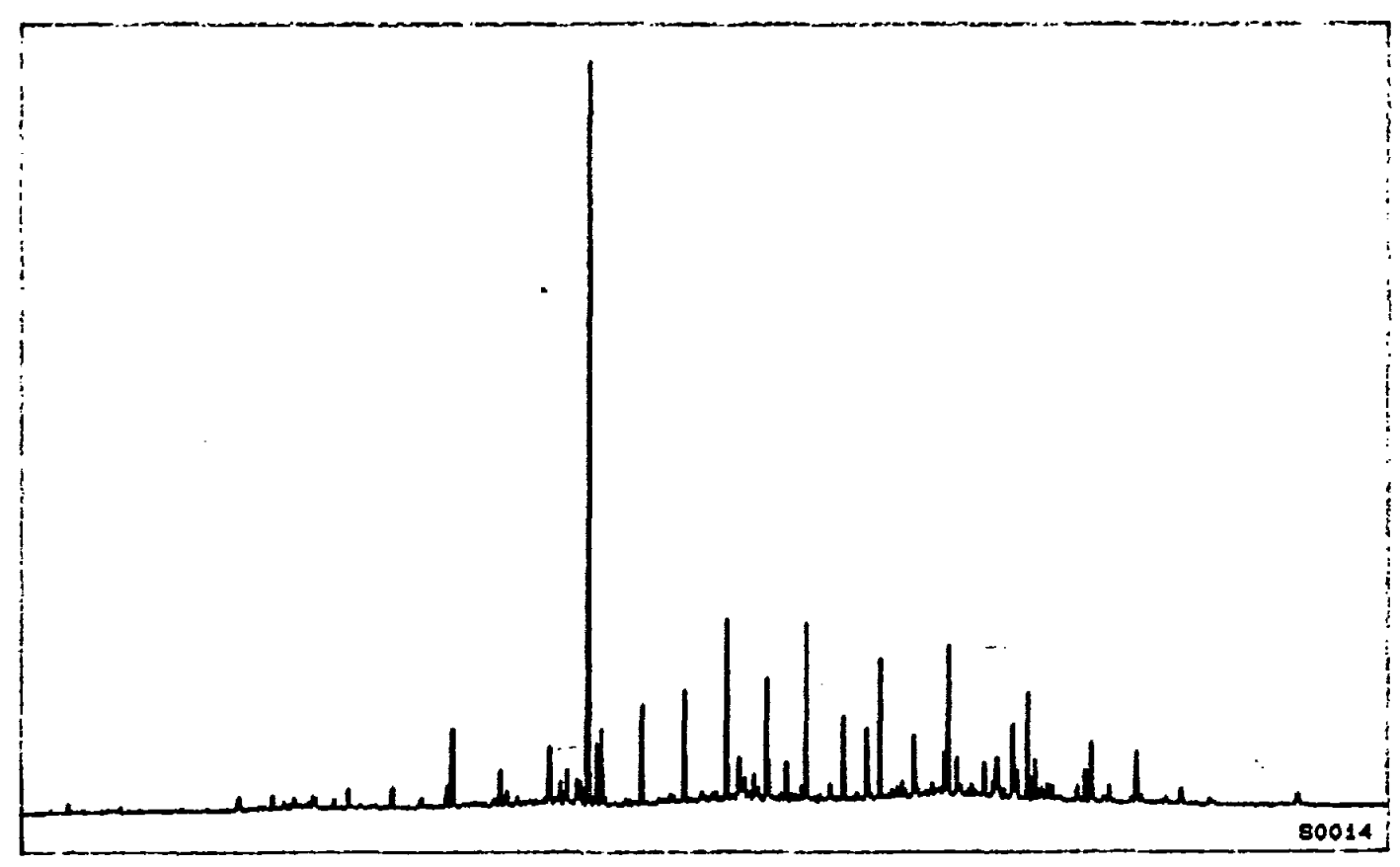

$1014.98 \mathrm{~m}$ (CUT)

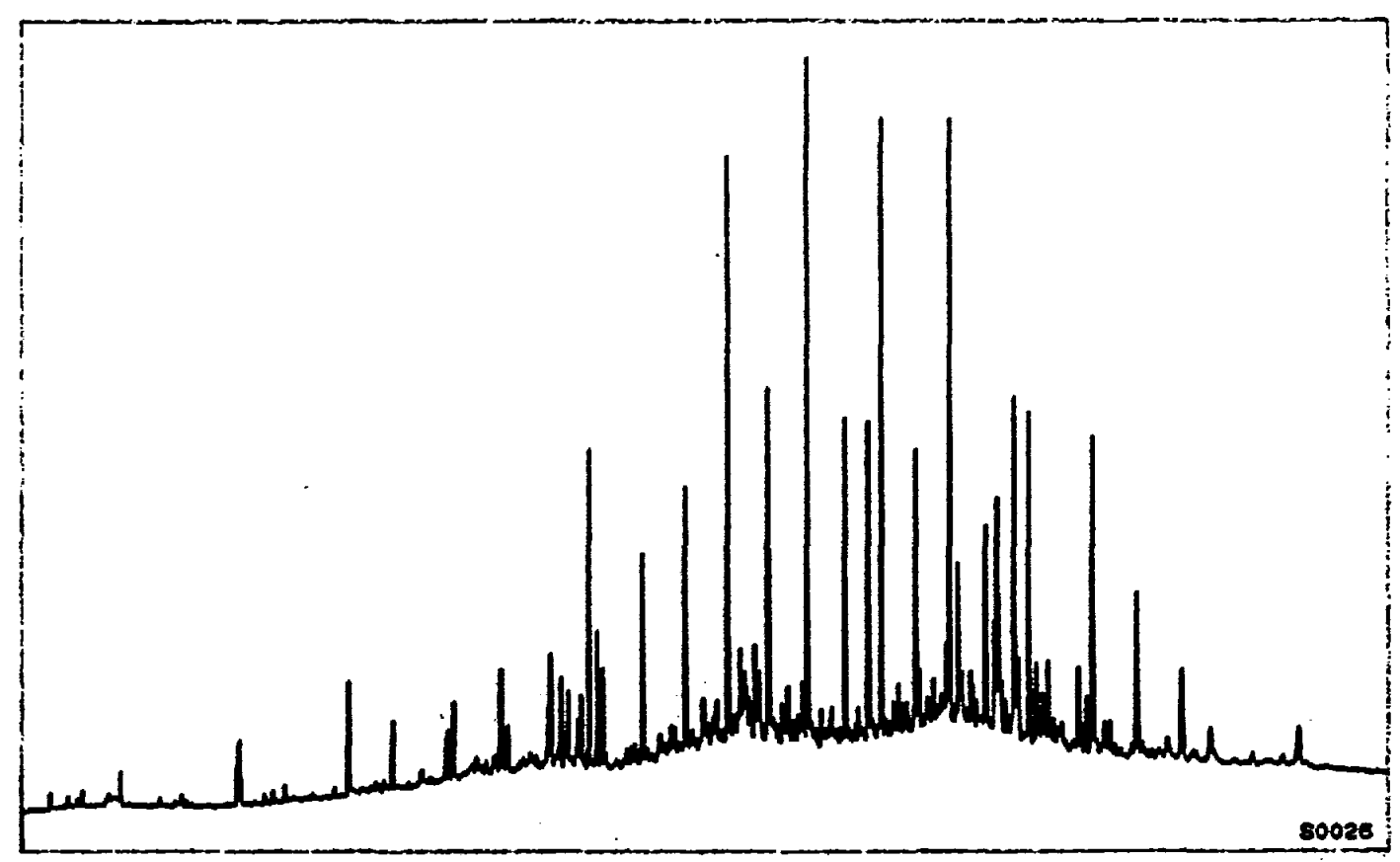

$1243.58 \mathrm{~m}$ (CUT)

SAC FRACTION CHROMATOGRAMS

WELL NAME : HAMMERHEAD-1

GMC Data Report 116

Page $19 / 44$ 


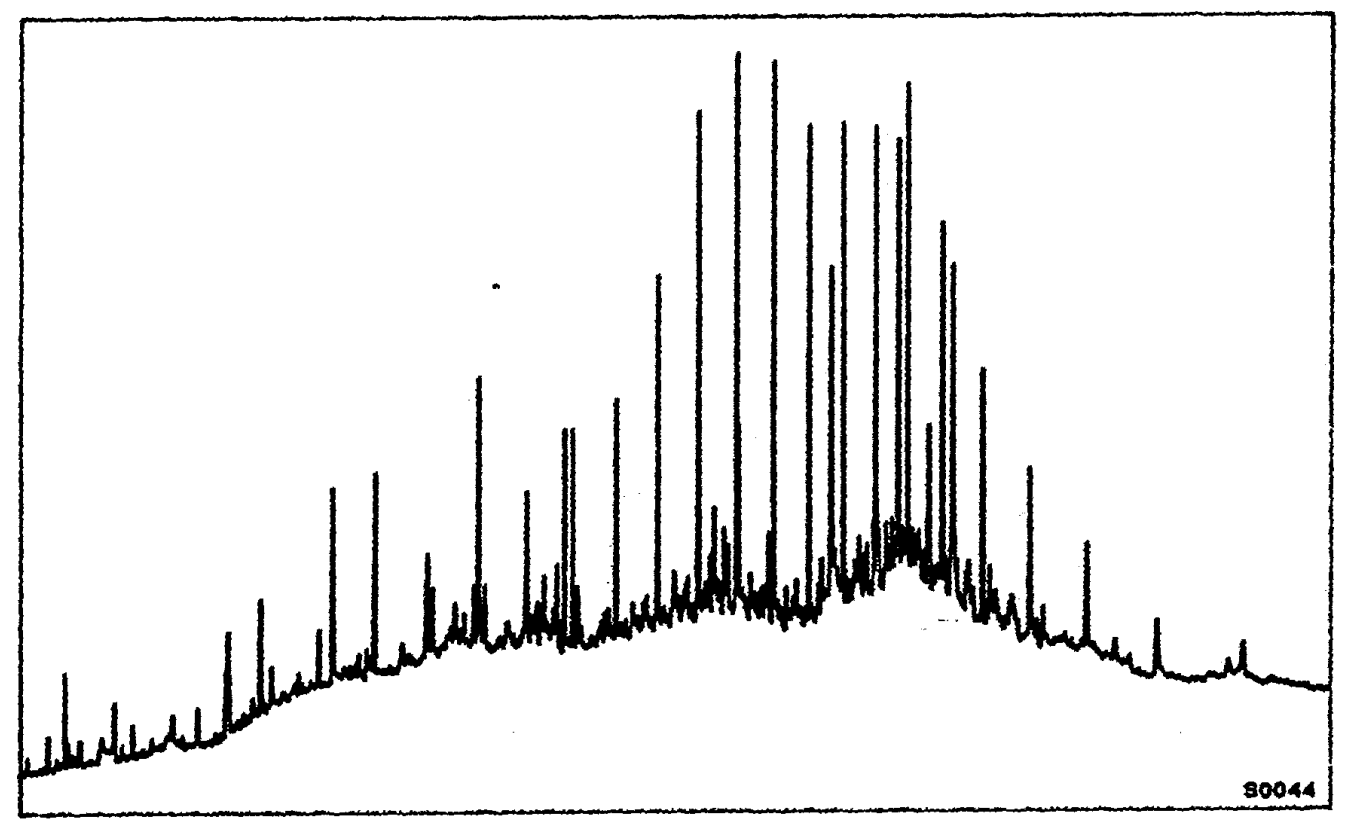

$1627.63 \mathrm{~m}$ (CUT)

6

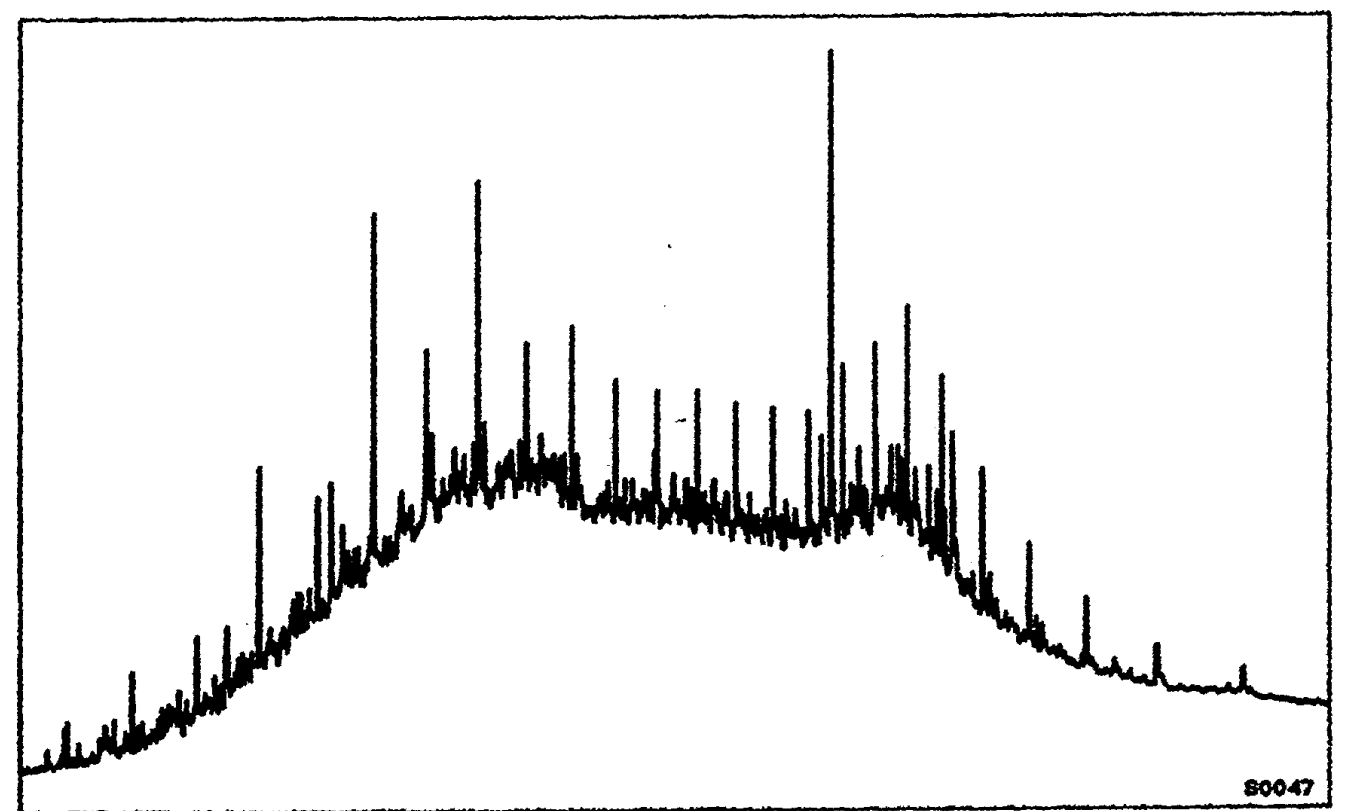

$1673.35 \mathrm{~m}$ (CUT)

SAC FRACTION CHROMATOGRAMS

WELL NAME : HAMMERHEAD-1

GMC Data Report 116

Page 20/44 


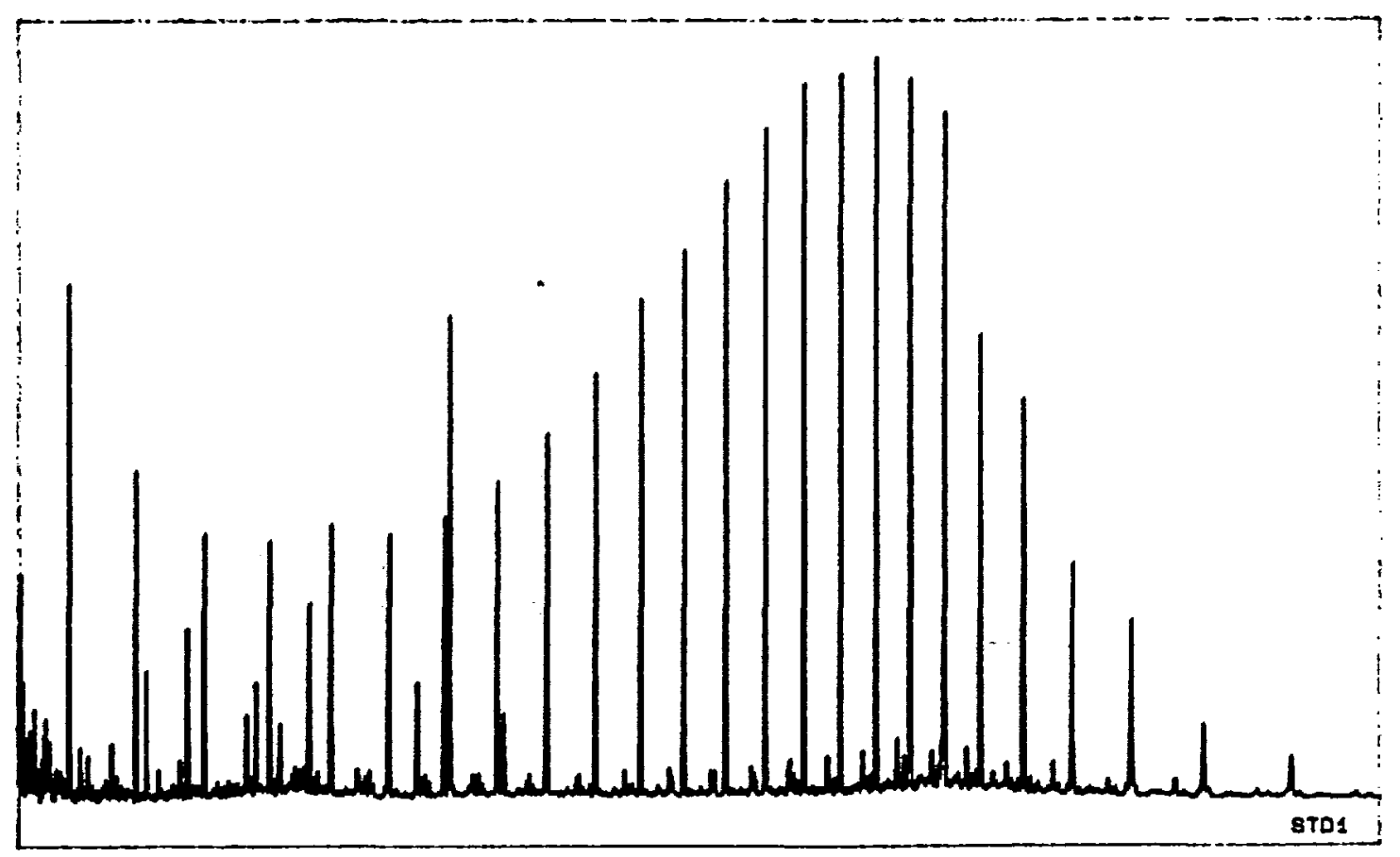

STANDARD

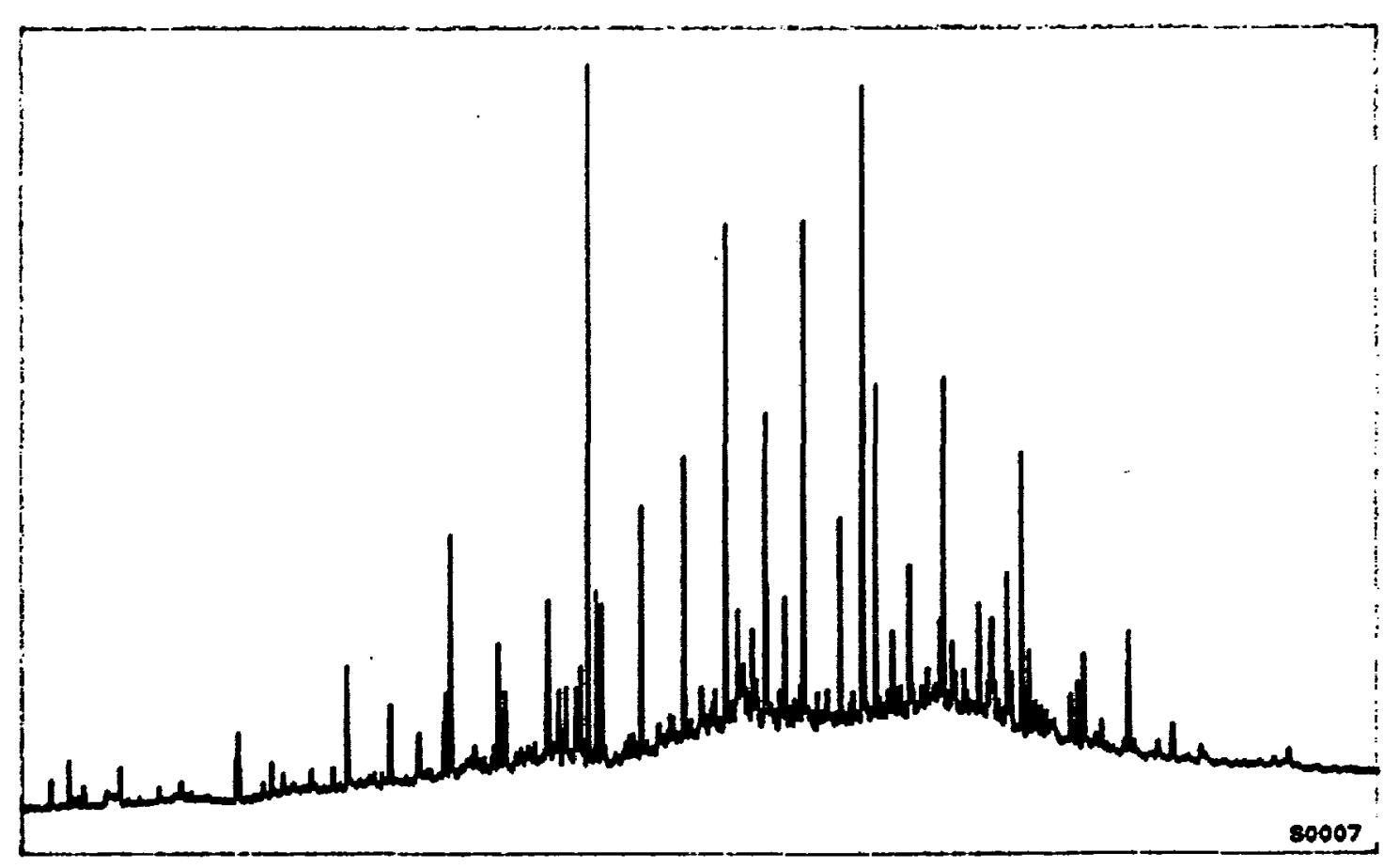

$941.83 \mathrm{~m}$ (CUT)

SAC FRACTION CHAOMATOGRAMS

WELL NAME : HAMMERHEAD-1

GMC Data Report 116

Page 21/44 


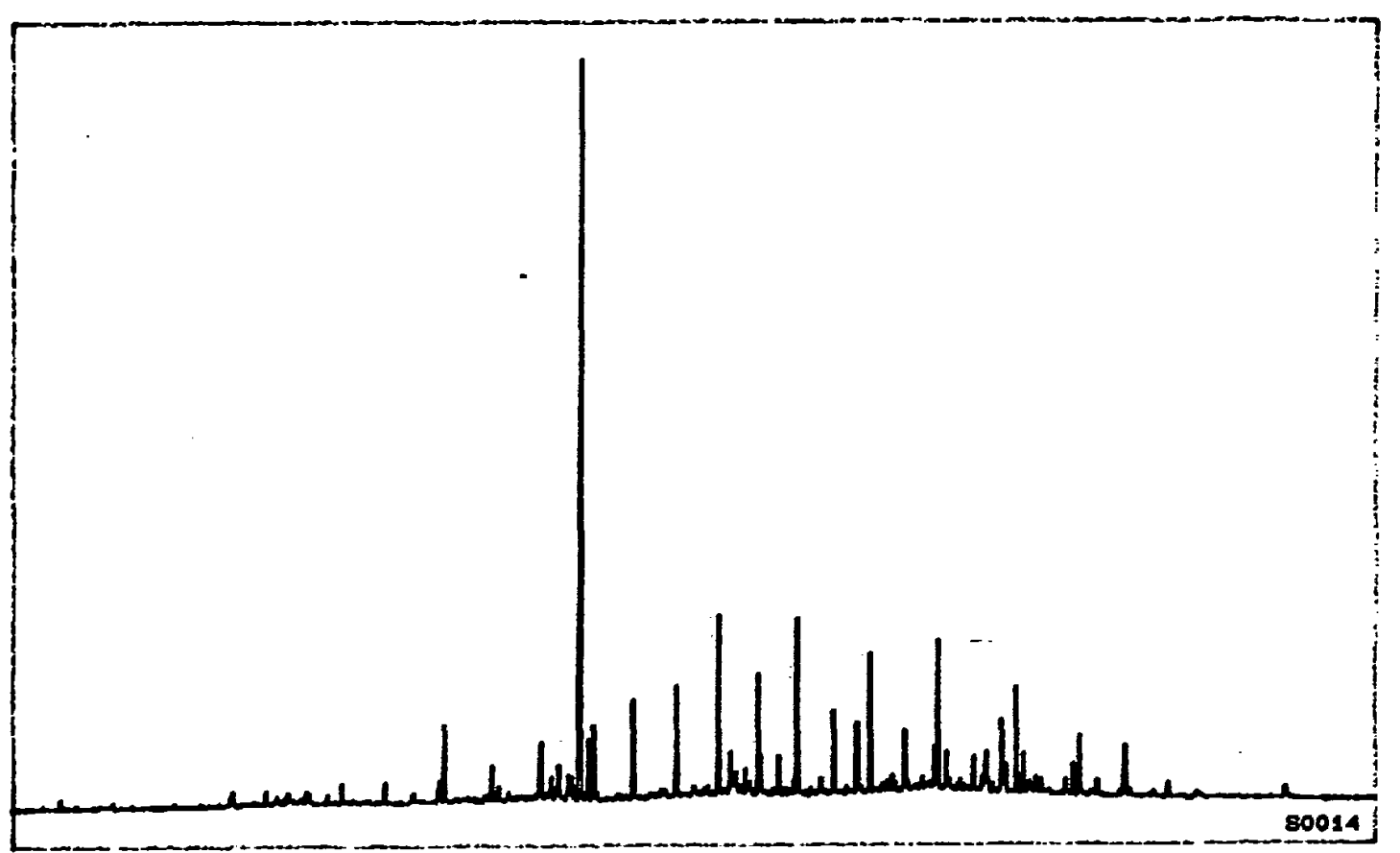

$1014.98 \mathrm{~m}$ (CUT)
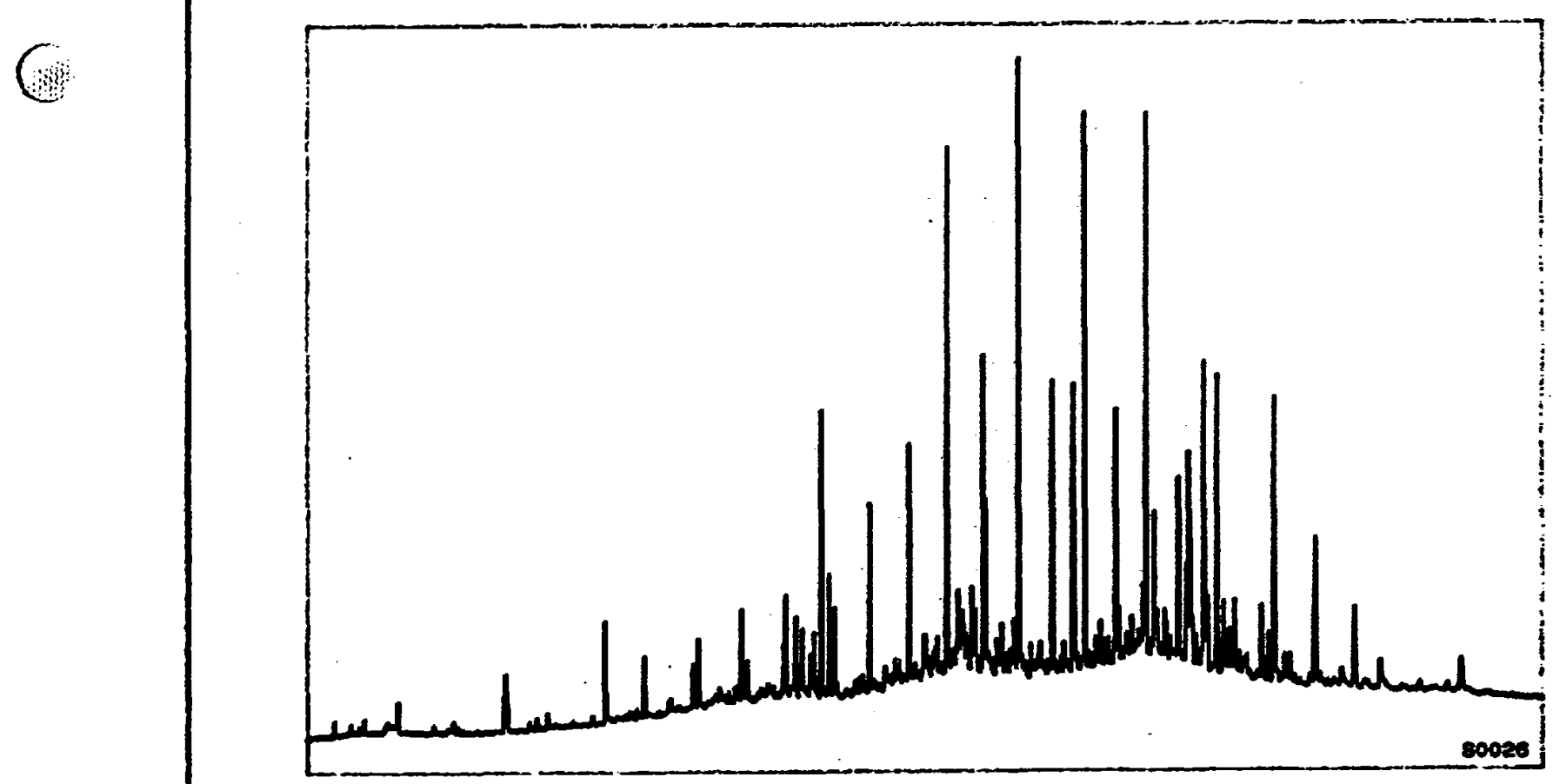

1243.58m (CUT)

SAC FRACTION CHROMATOGRAMS

WELL NAME : HAMMERHEAD-1 


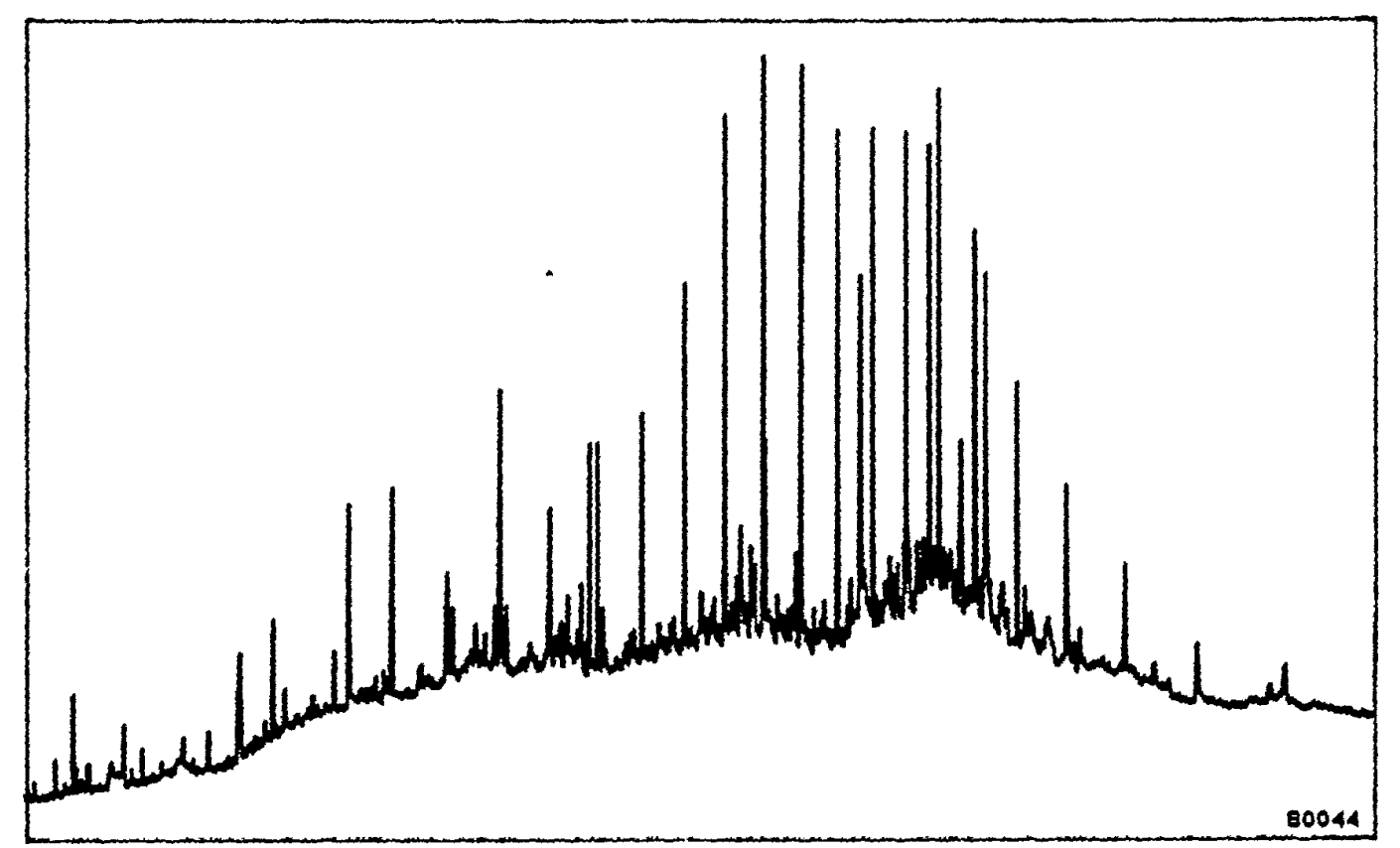

$1627.63 \mathrm{~m}$ (CUT)

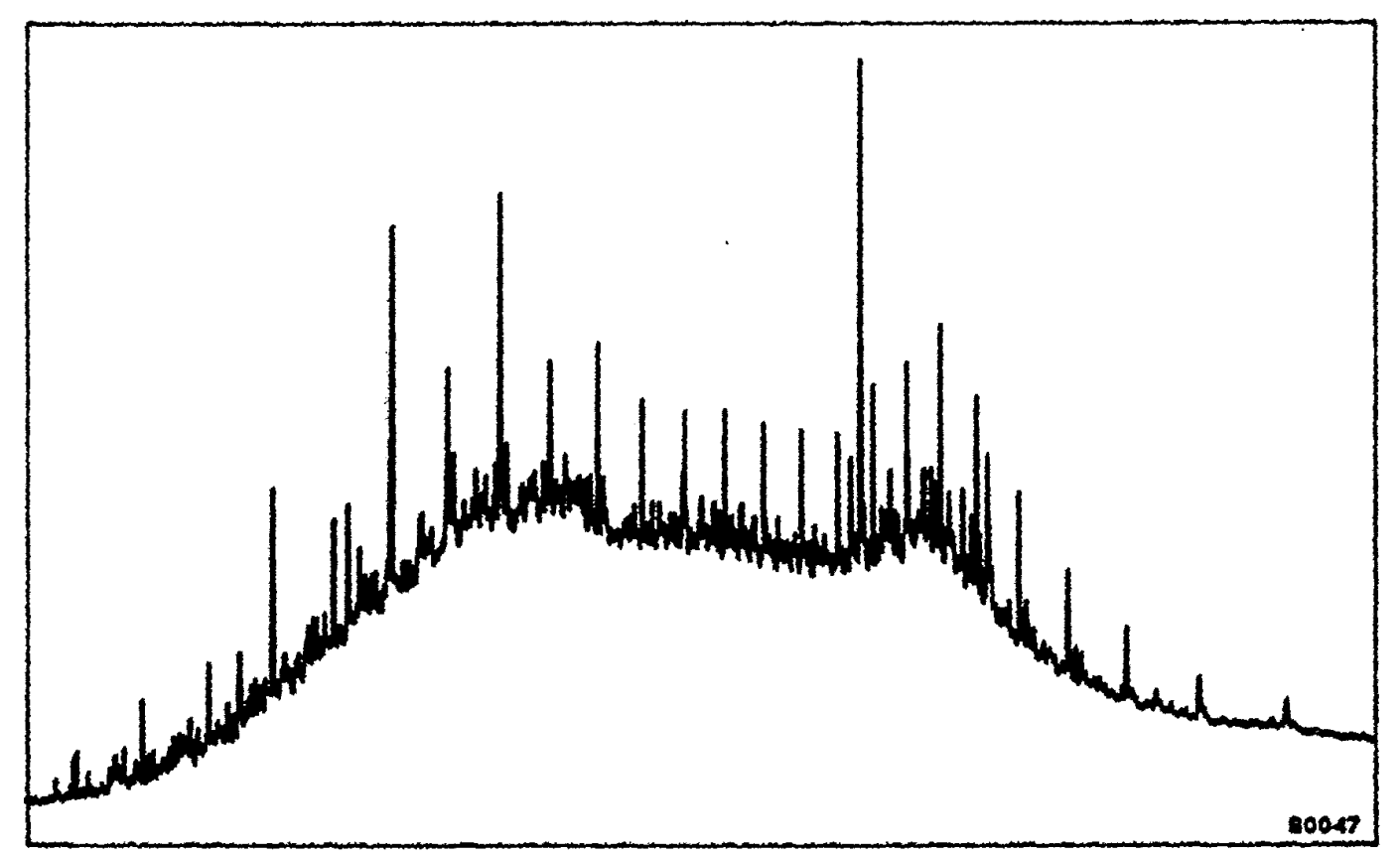

$1673.35 \mathrm{~m}$ (CUT)

SAC FAACTION CHROMATOGAAMS

WELL NAME : HAMMERHEAD-1

GMC Data Report 116

Page $23 / 44$

GCB ref. B8125ED007 


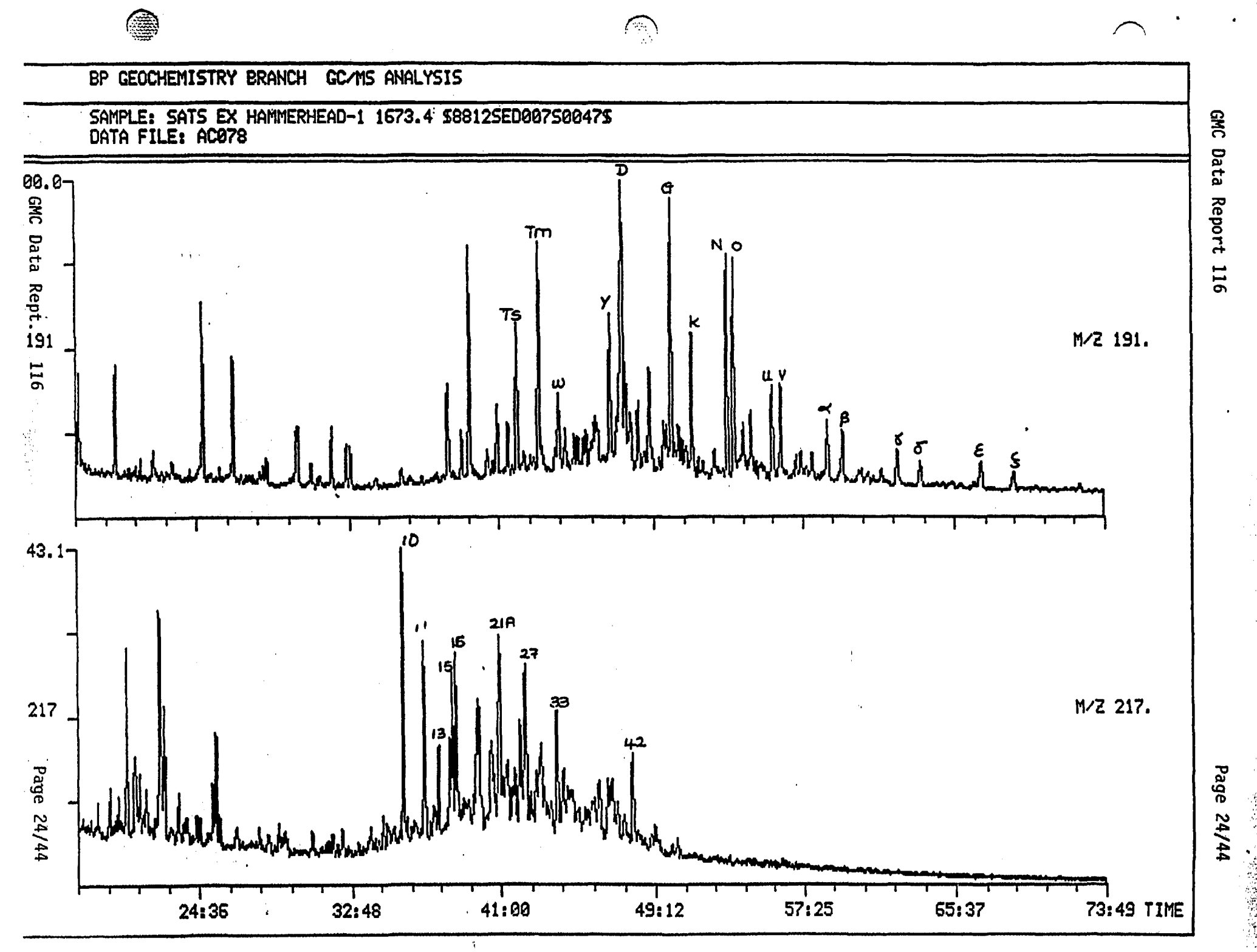




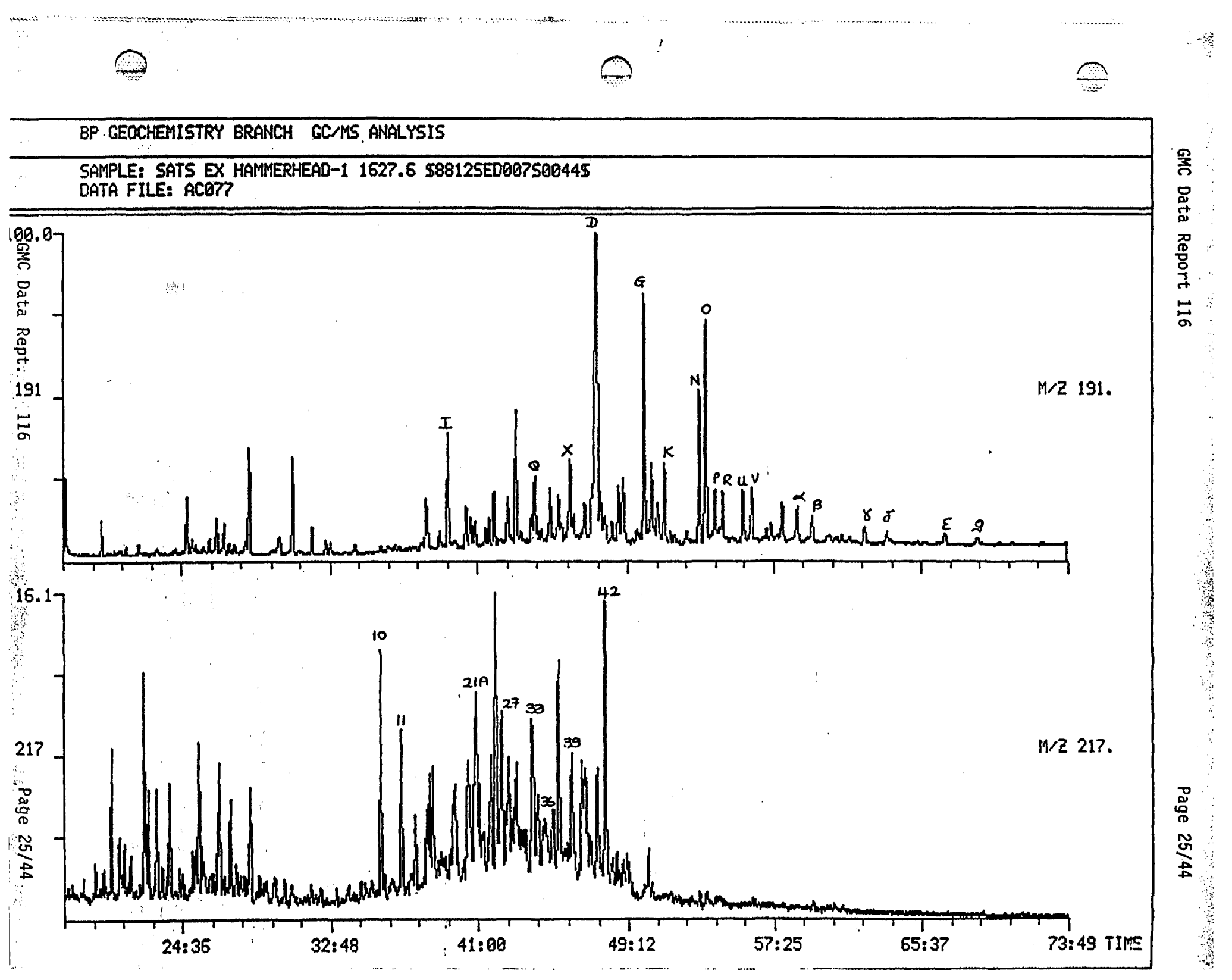




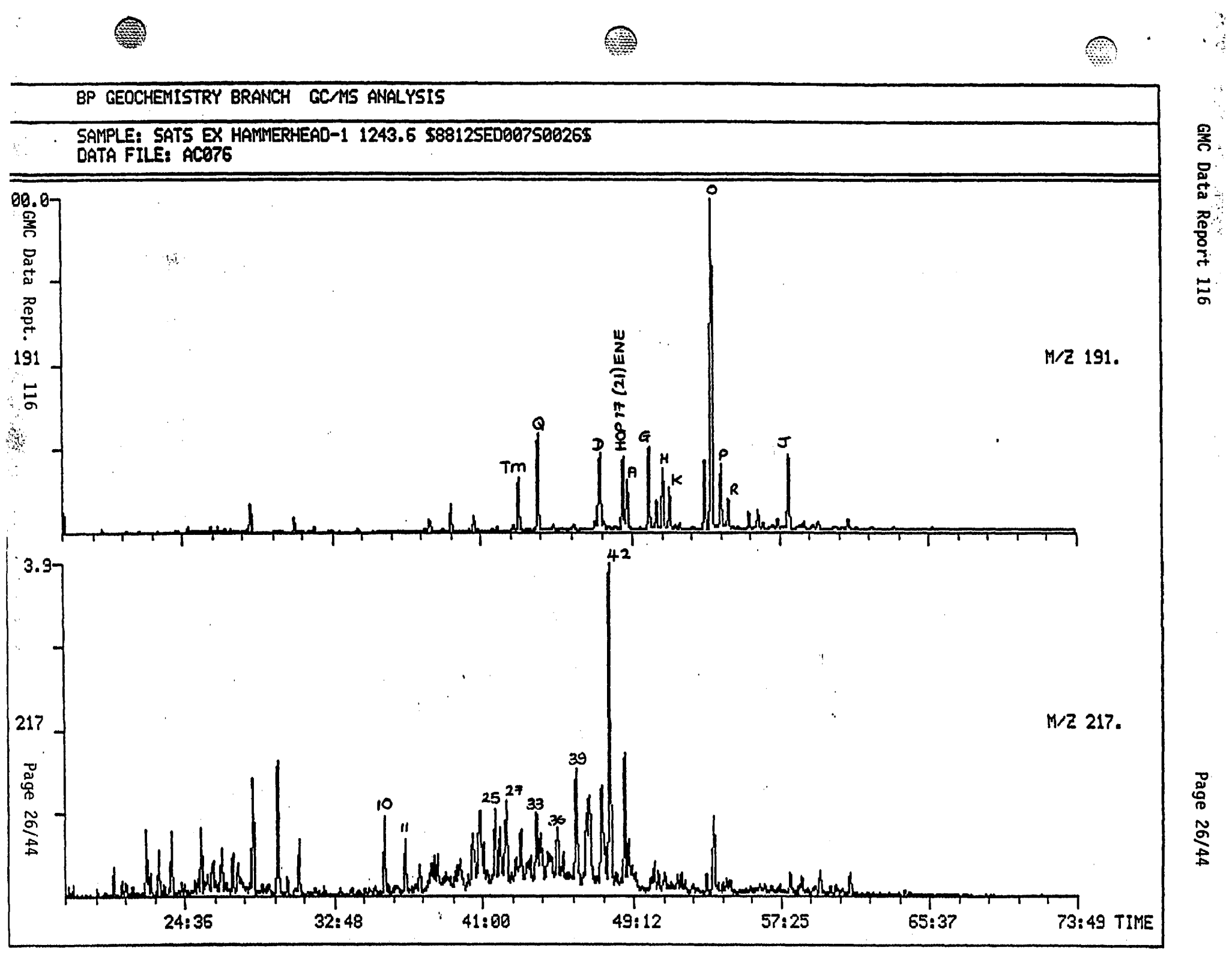




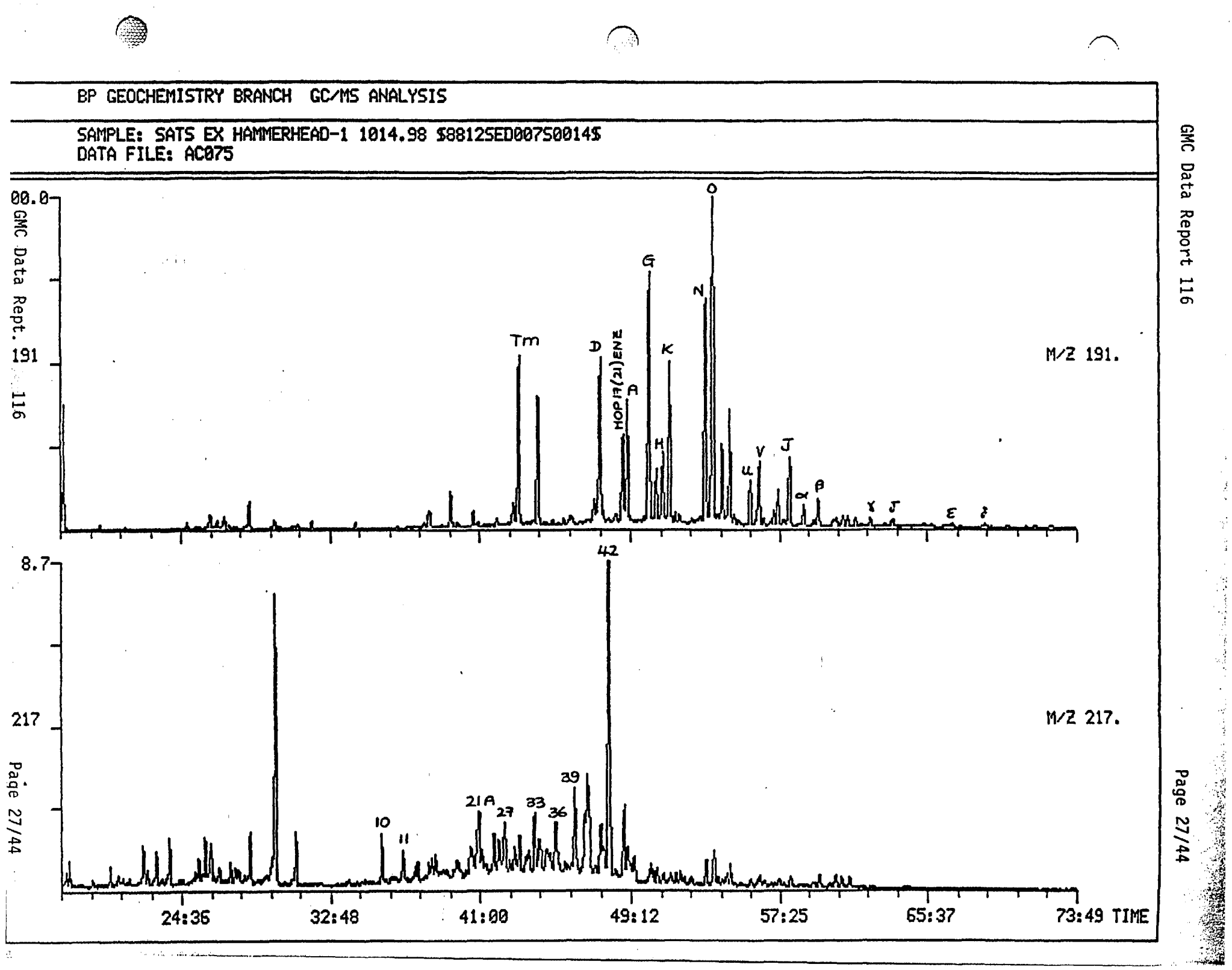




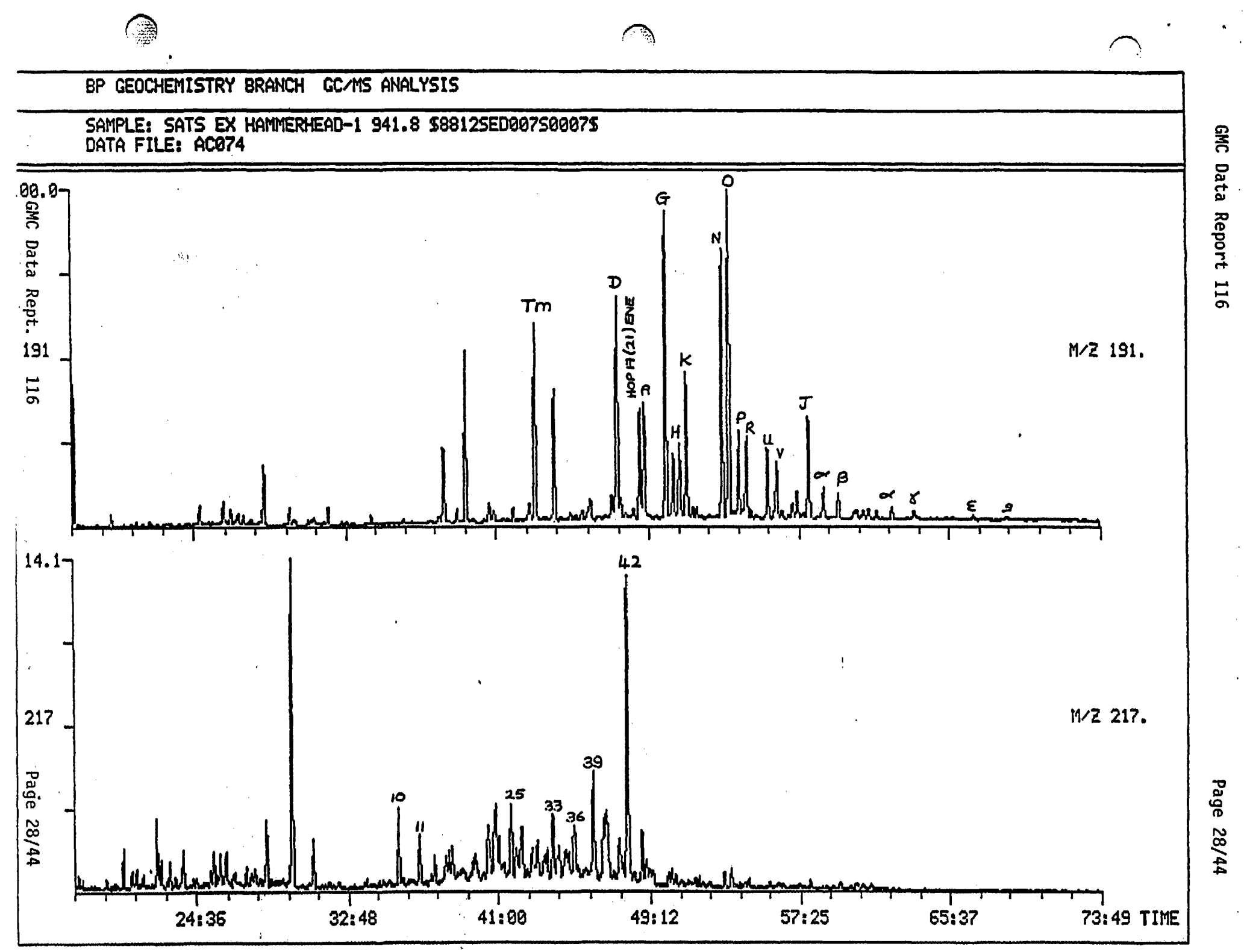




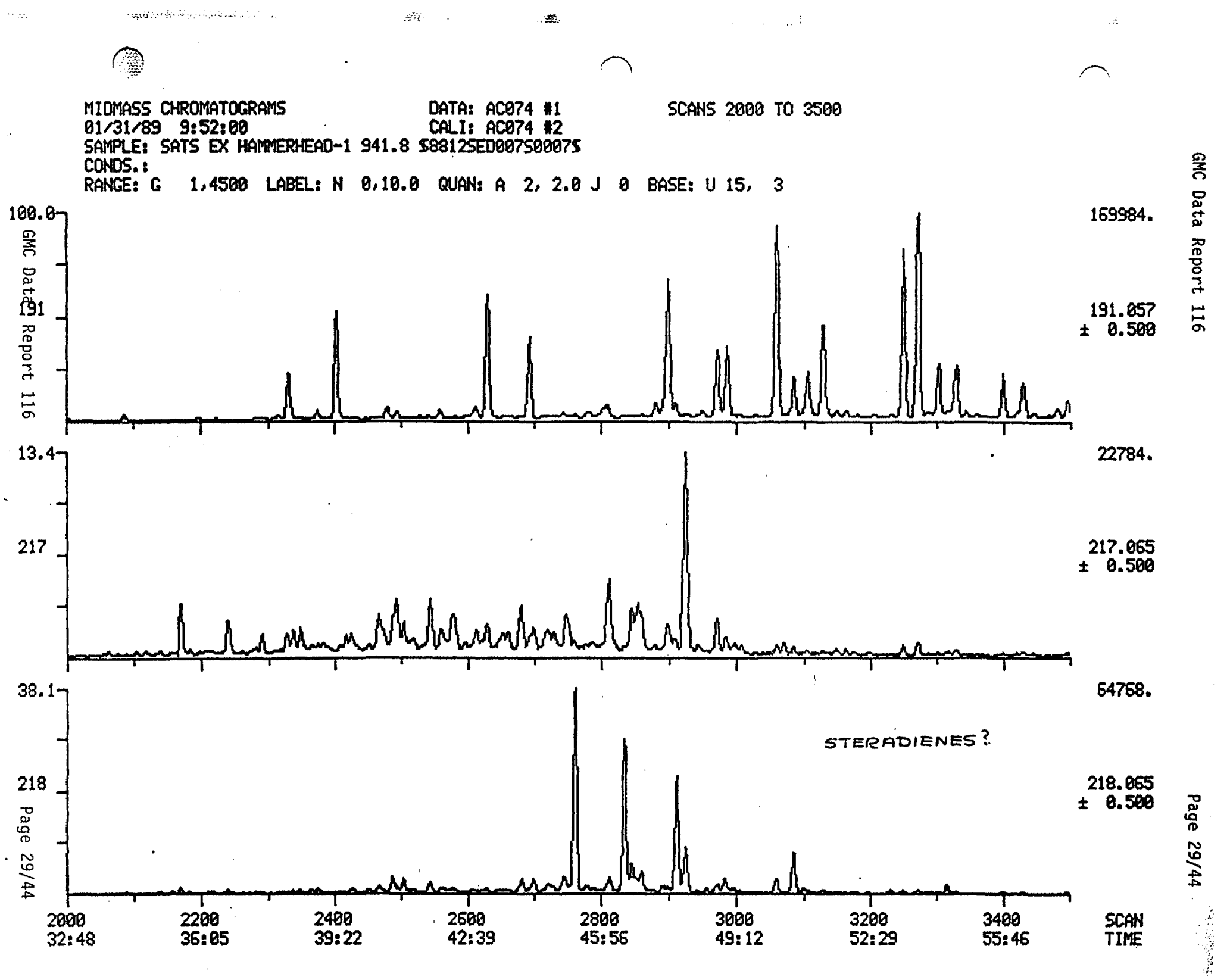




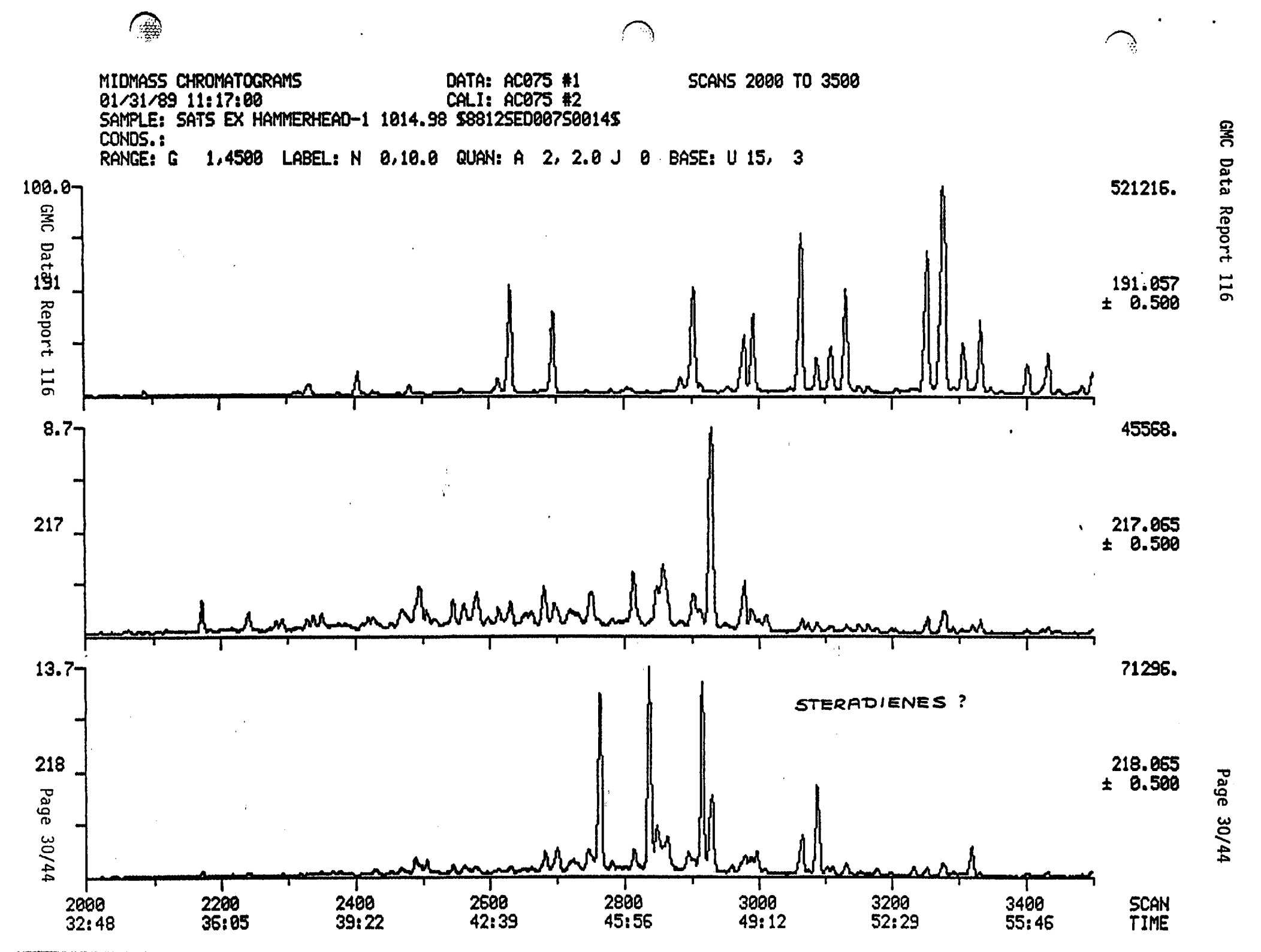




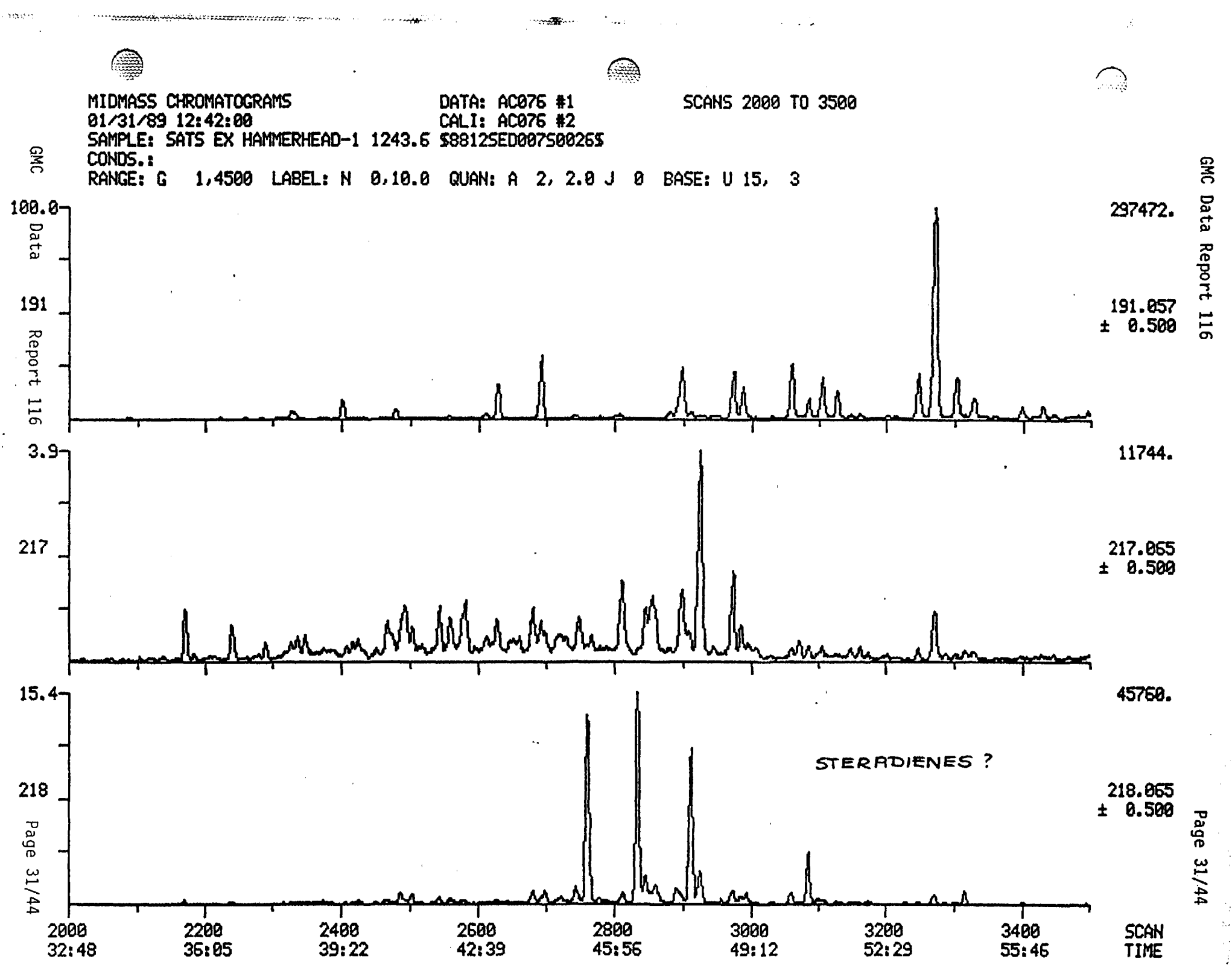




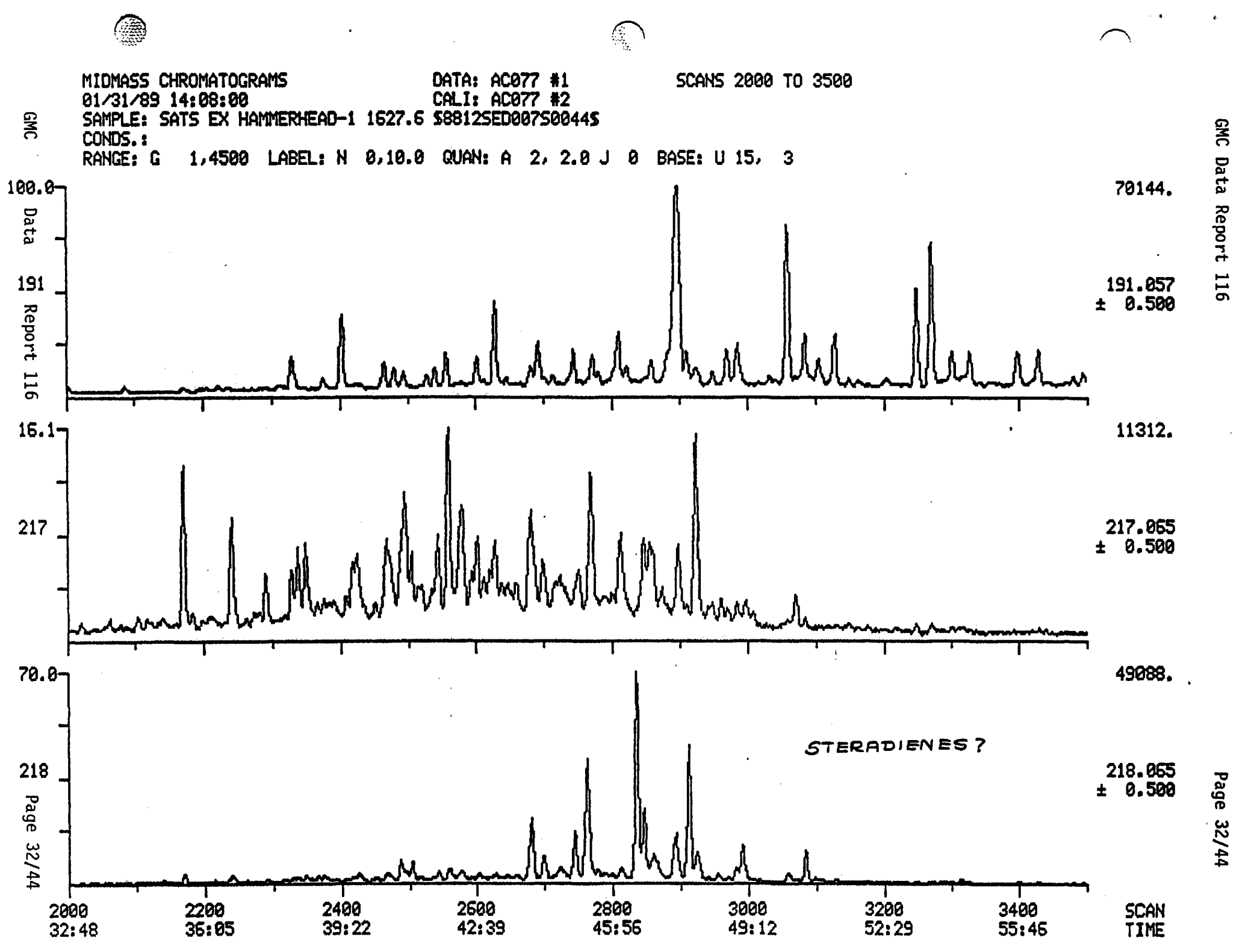




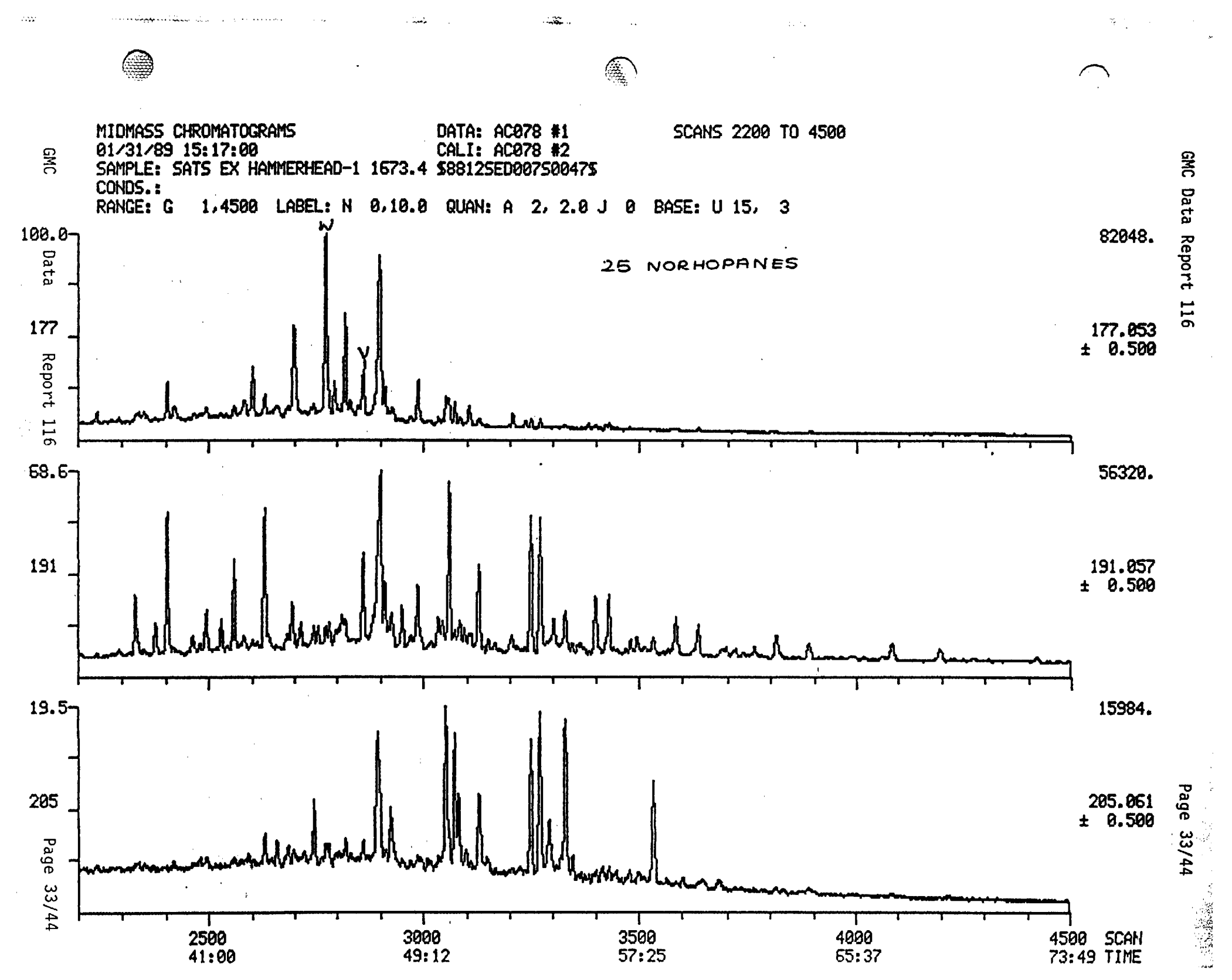




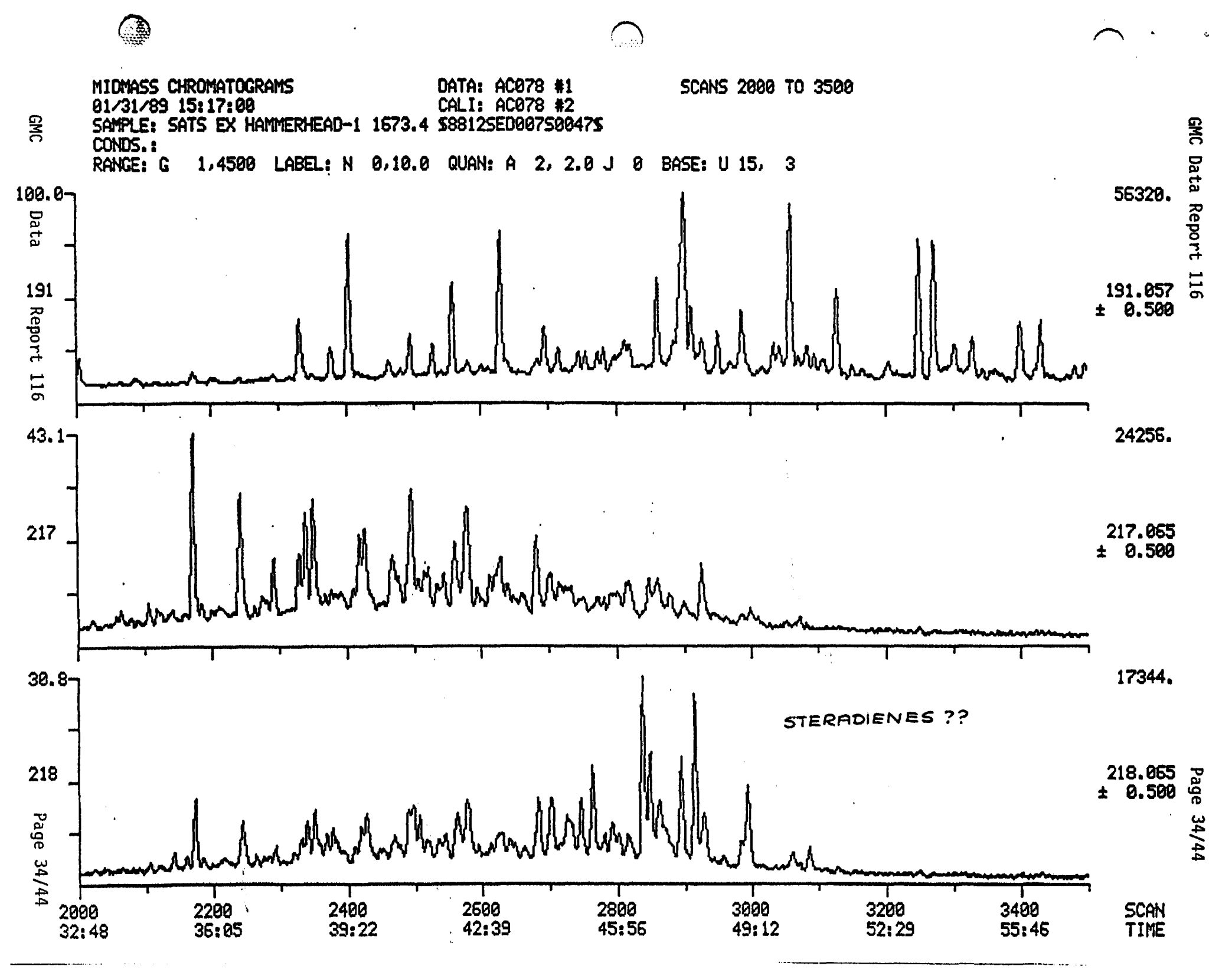




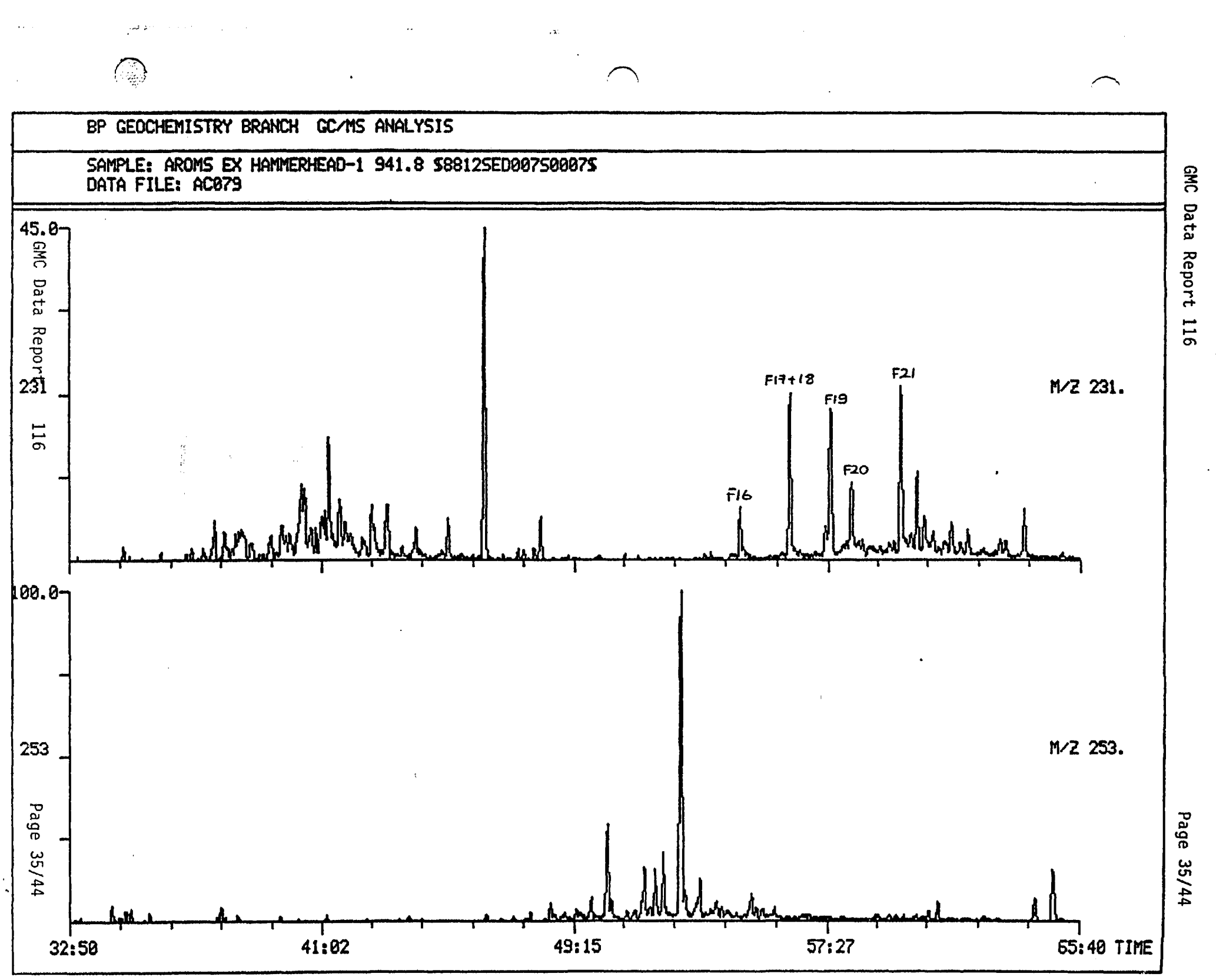




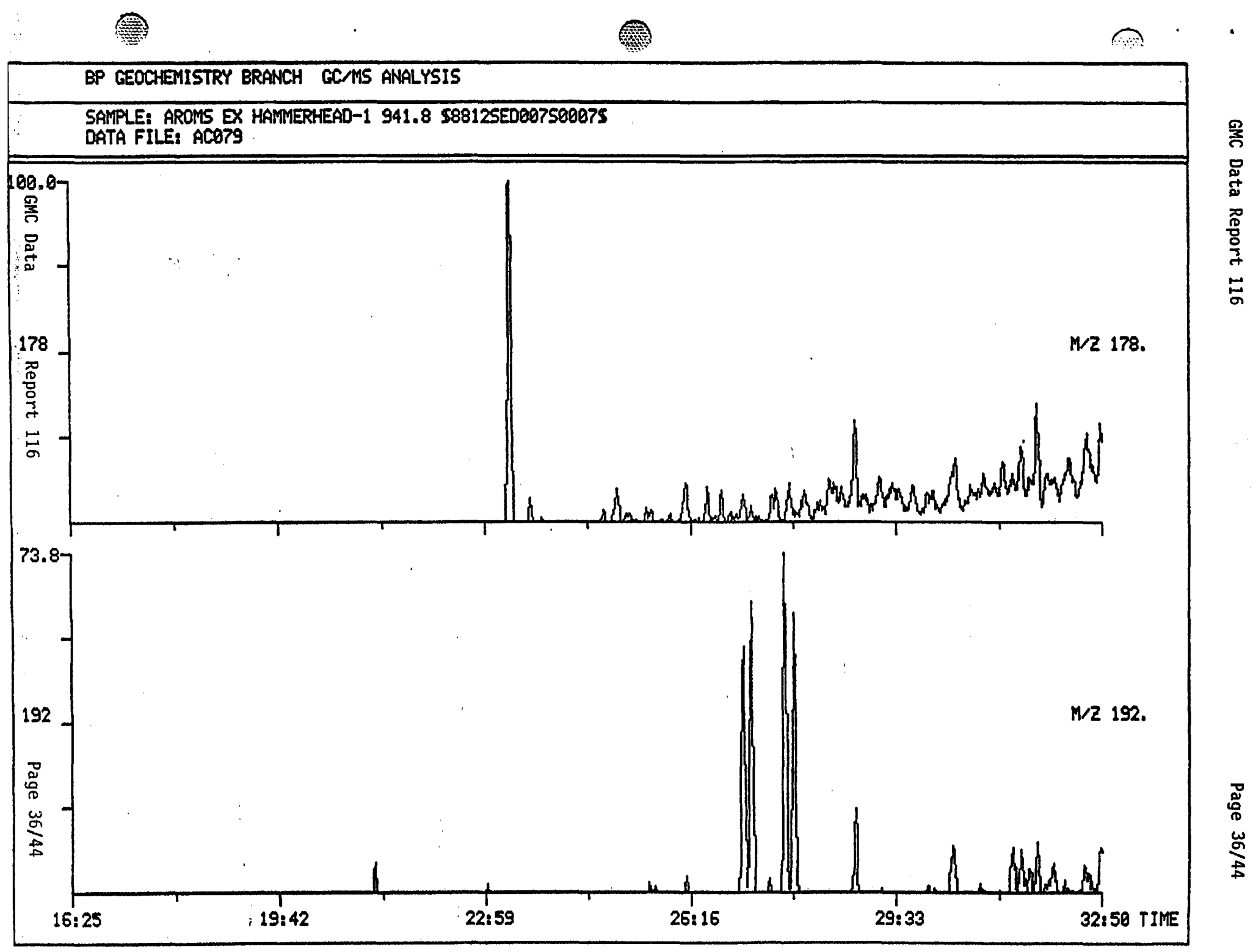




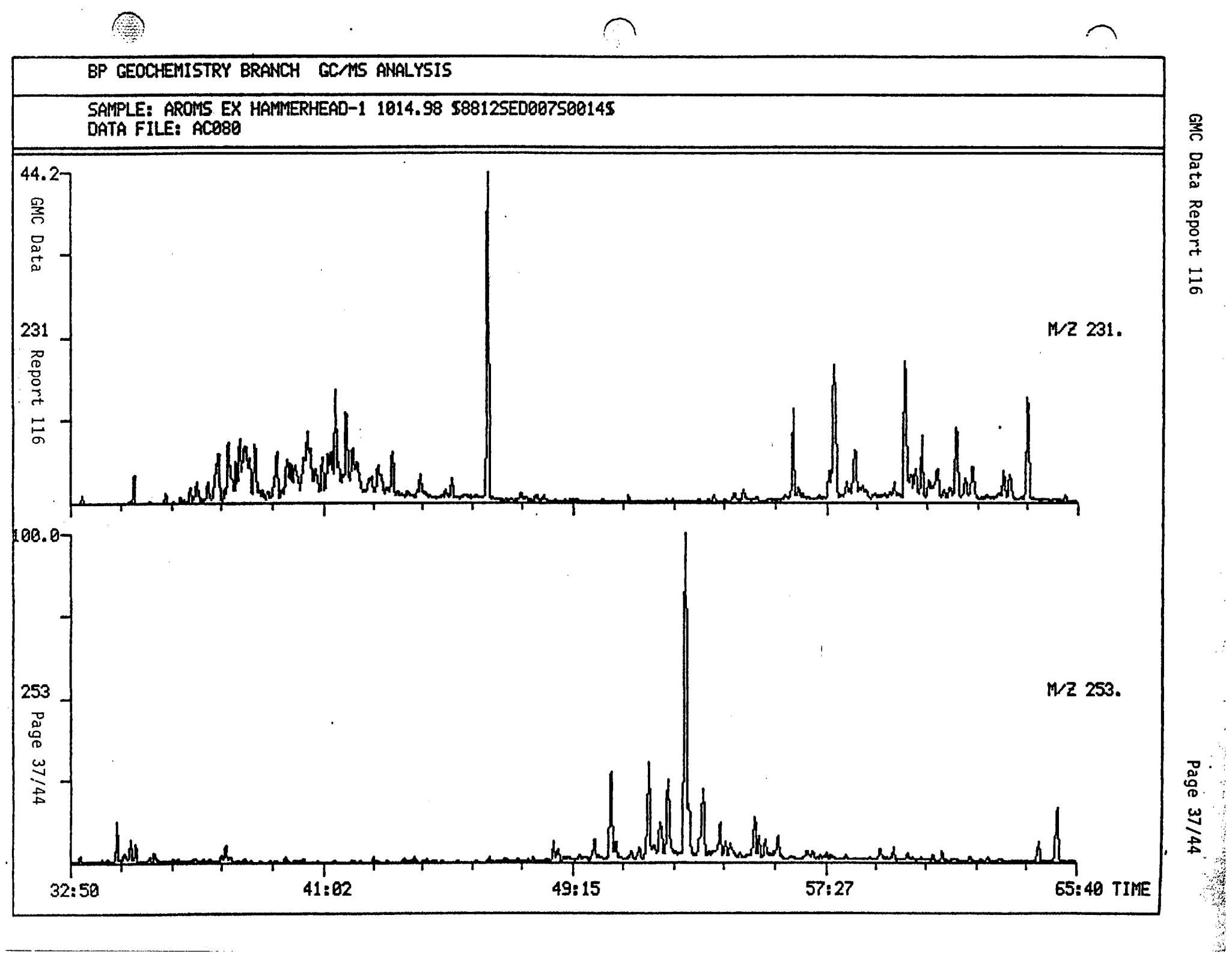




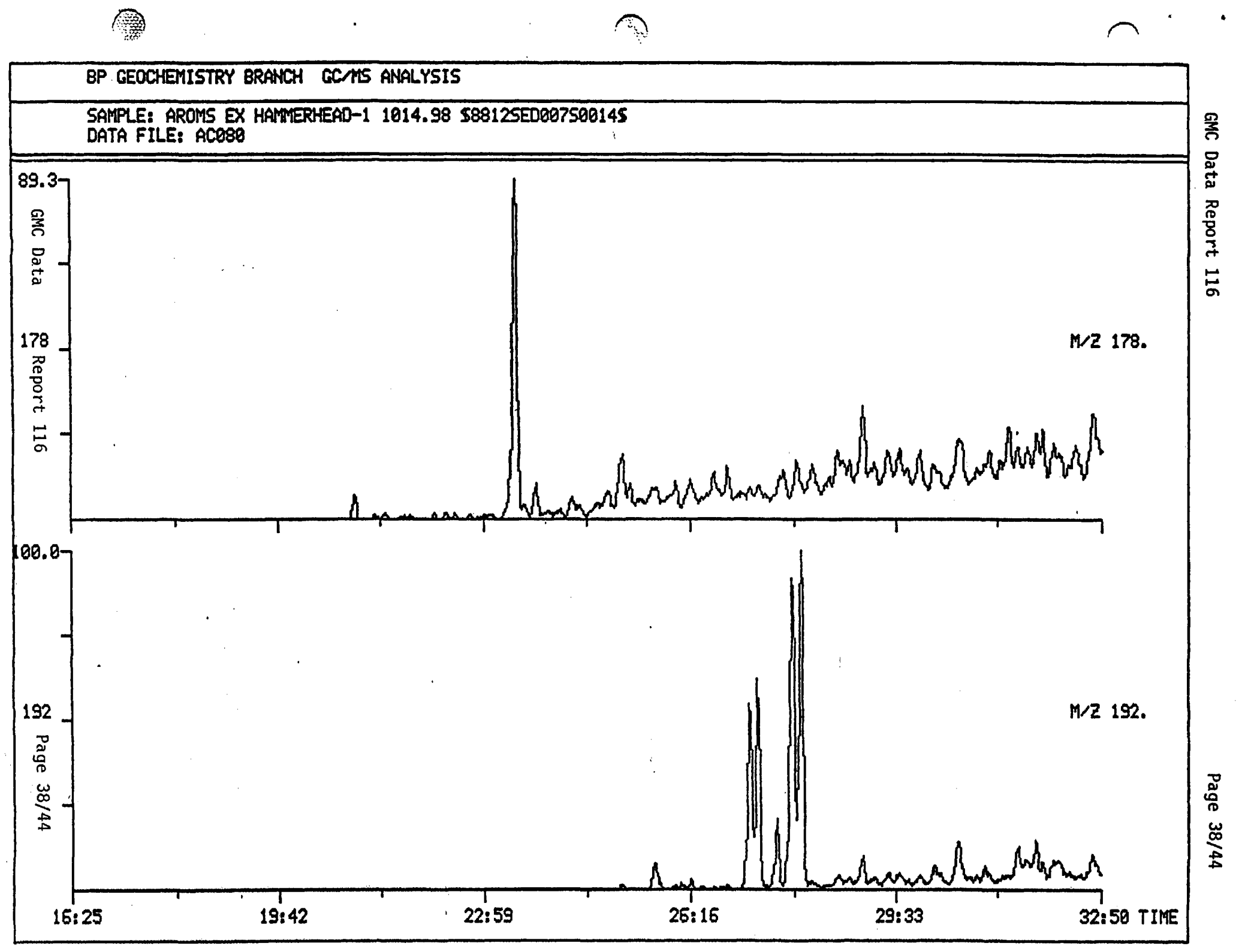




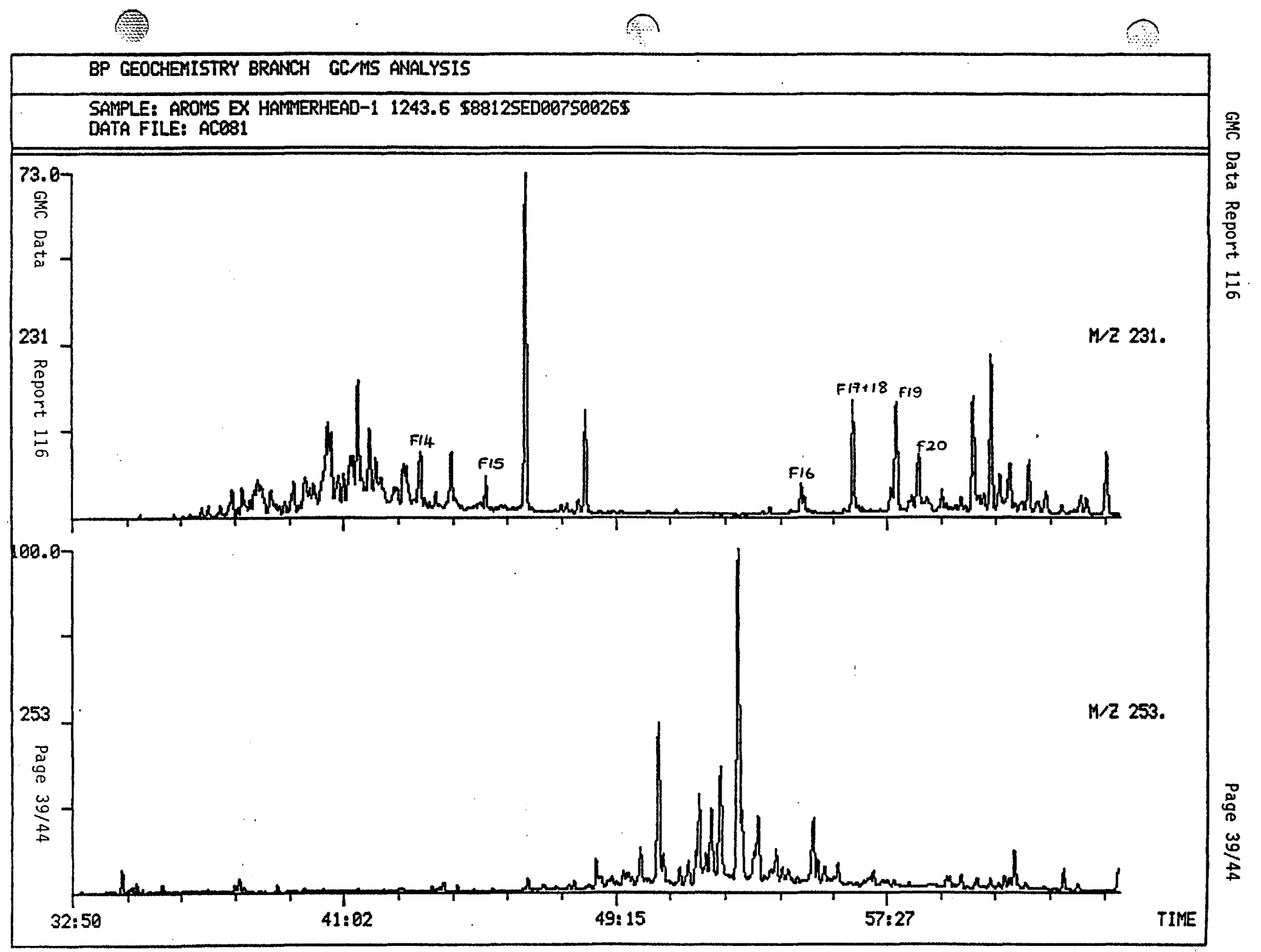




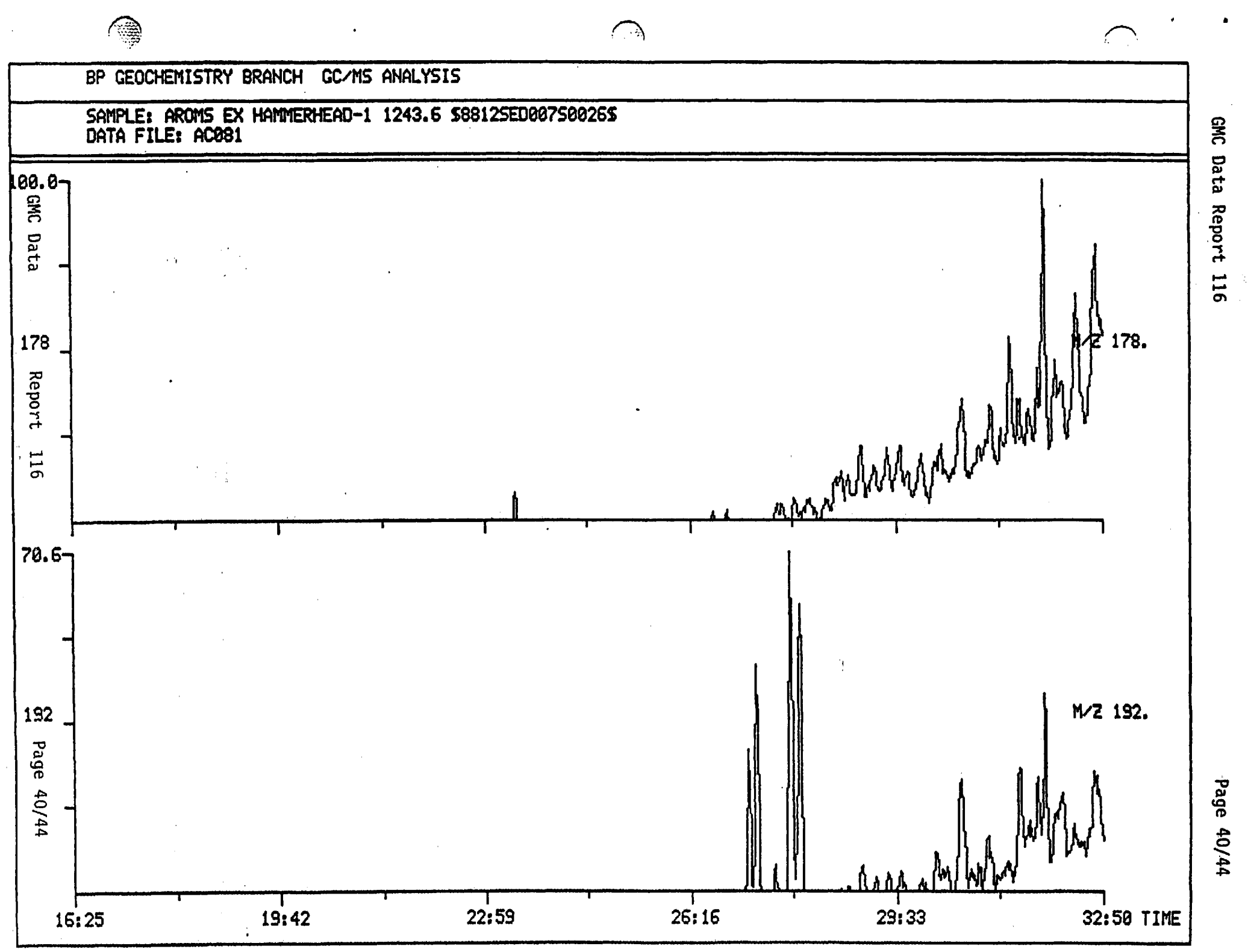


PEOCHEMISTRY BRANCH GCASS ANALYSIS

SAMPLE: AROMS EX HAMMERHEAD-1 1627.6 \$8812SED007568445

DATA FILE: ACO82

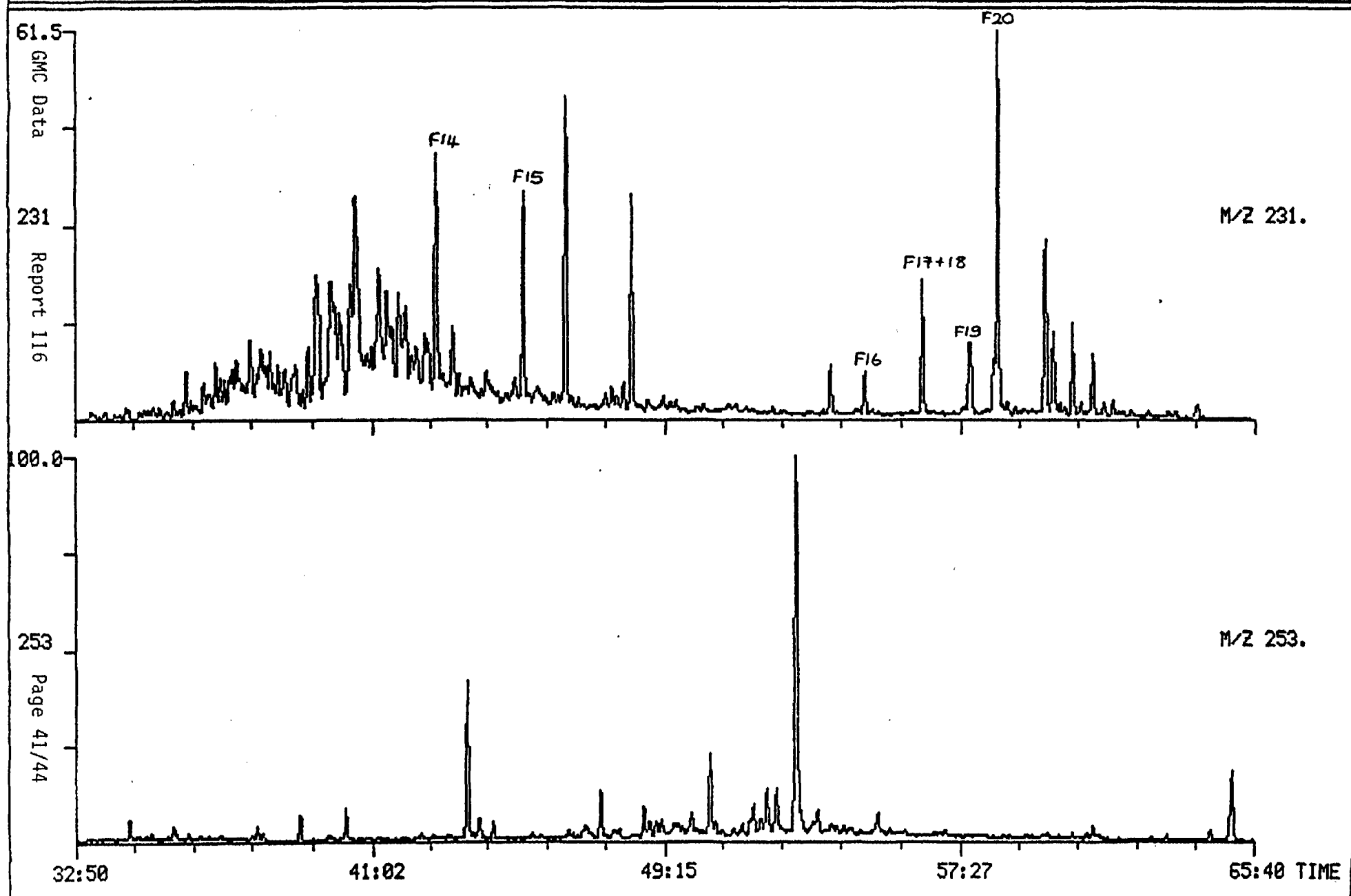




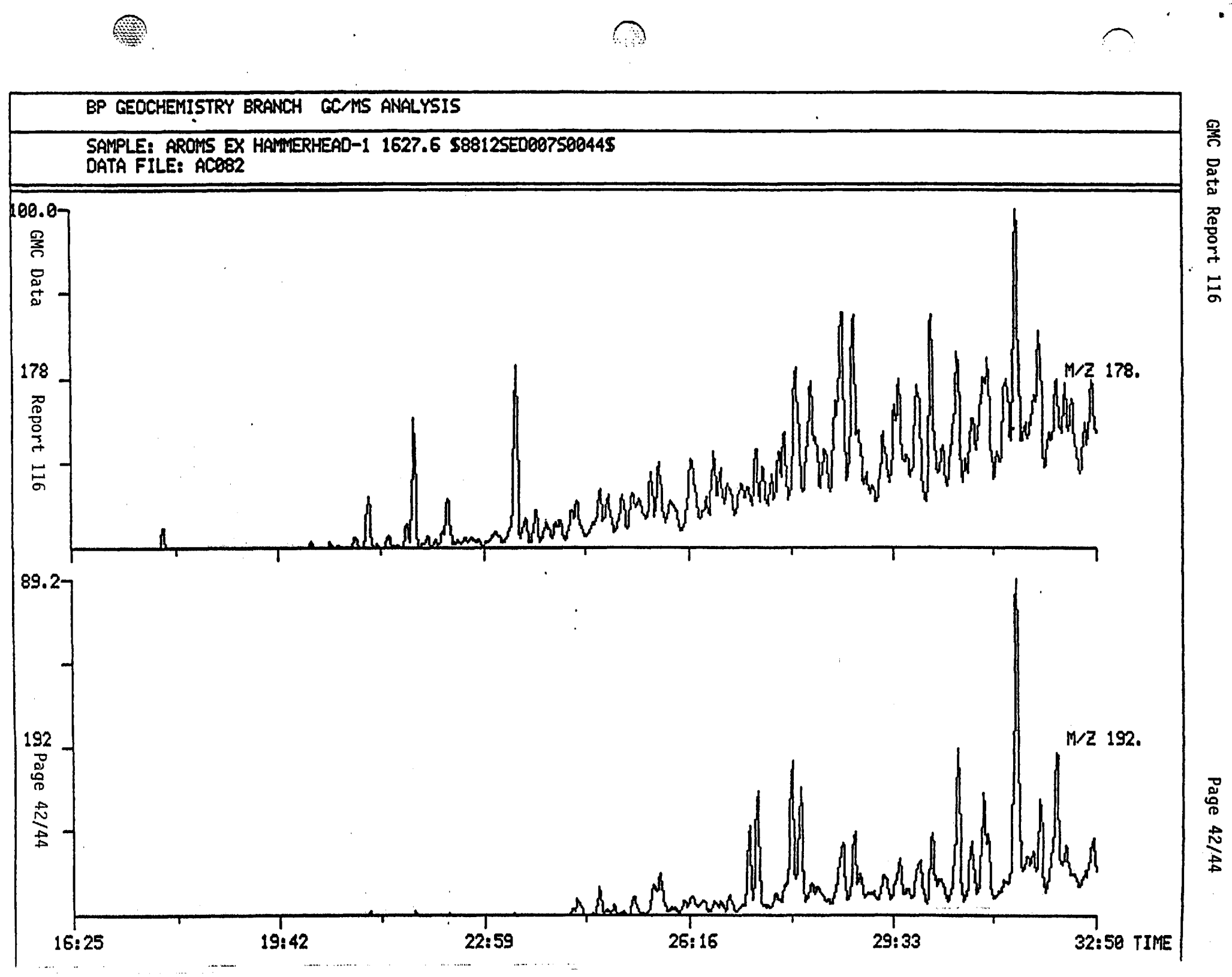




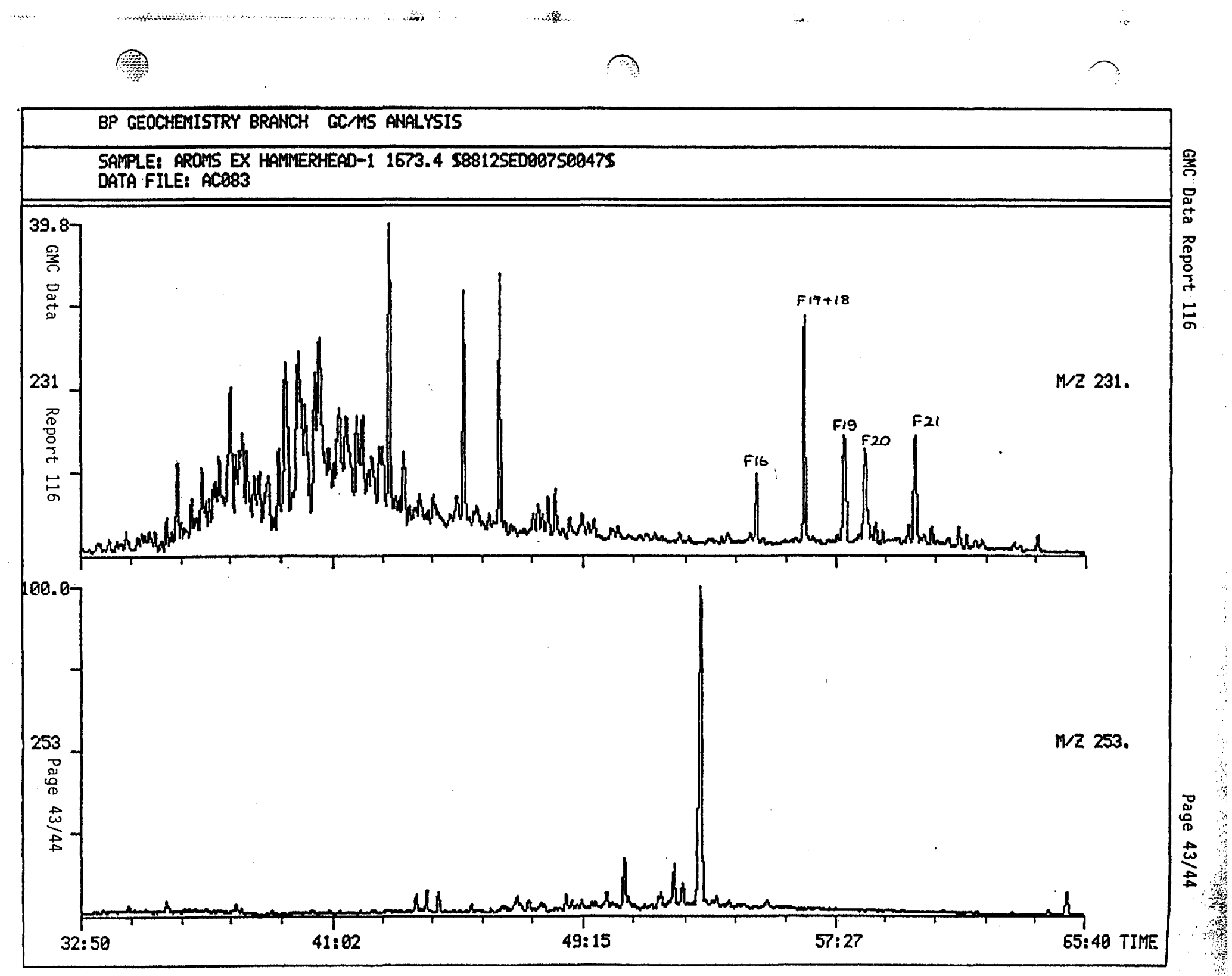




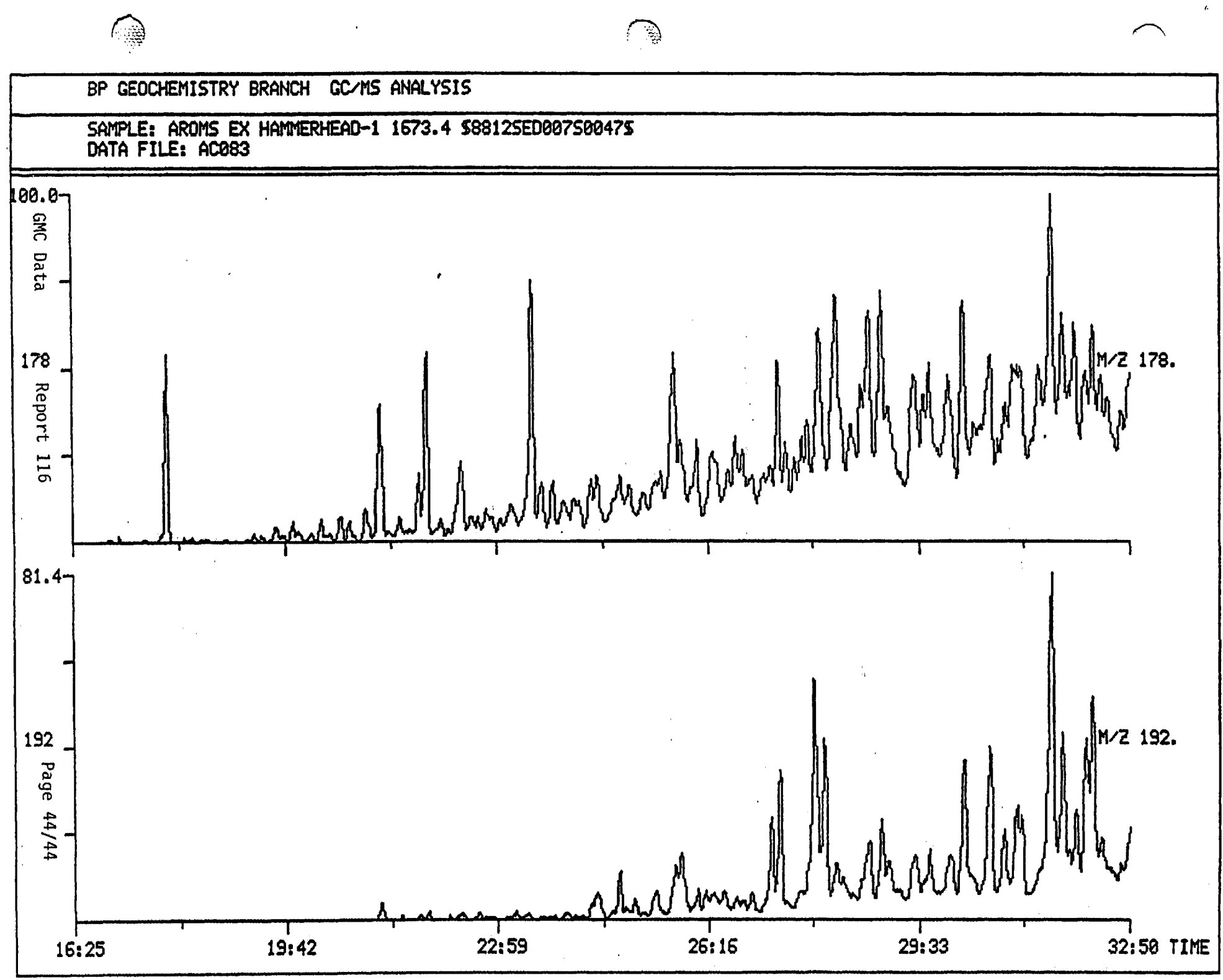


Geochemical data of cuttings and sidewall cores from the Union 0 il Company OCS Y-0849-1 (Hammerhead No. 1) well

Supplement A to the Geologic Materials Center Data Report No. 116

This material is from the U. S. Minerals Management Service

Total of 4 pages in report (A-1 through $A-4)$ 
December 3, 1985

Mr. Brian F. Schoof

United States Department of Interior

Minerals Management Service

Alaska OCS Region

P.D. Box 101159

Anchorage, AK 99510

Dear Mr. Schoof:

Attached are the final results of geochemical work on samples from Union, OCS $Y$-0849非 1 . Table 1 includes data from cuttings samples. Table 2 includes

sidewall core data. The contents of these two tables are described as follows:

$\begin{array}{ll}\text { Depth } & \text { Measured depth, in feet } \\ \text { TOC } & \text { Total organic carbon, in percent } \\ \text { S1 } & \text { Sl Rockeval yields in } \mathrm{mg} / \mathrm{g} \\ \text { S2 } & \text { S2 Rockeval yields in } \mathrm{mg} / \mathrm{g} \\ \text { S3 } & \text { i3 Rockeval yields in } \mathrm{mg} / \mathrm{g} \\ \text { Tmax } & \text { iemperature at S2 maximum, in degrees C } \\ \text { VR } & \text { Vitrinite reflectance, measured on whole rock (\%) }\end{array}$

These comprise all the geochemical logging data Union has concerning the rock samples from this well. We would be willing to answer questions concerning these data at anytime.

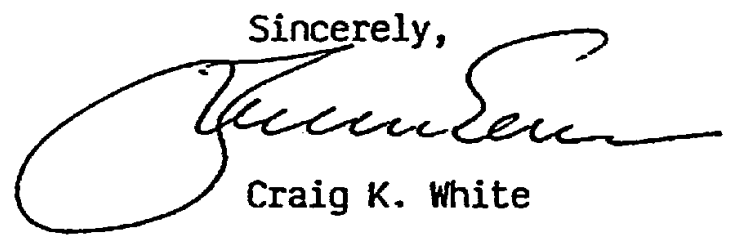

CKW: Ims

(2013e)

Attachment

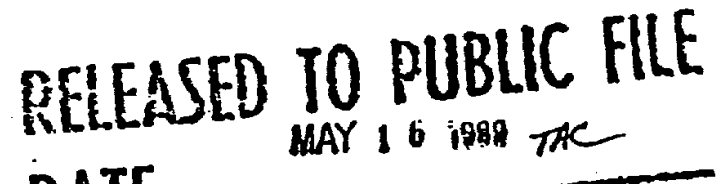
DATE 
GMC Data Report 116 : $:-$ Page A-2/4

$\because \because \cdots$ IUK.

TAELE 1

OCS-Y-\$4O-1 HELL, CUTTINGS SAMOLES

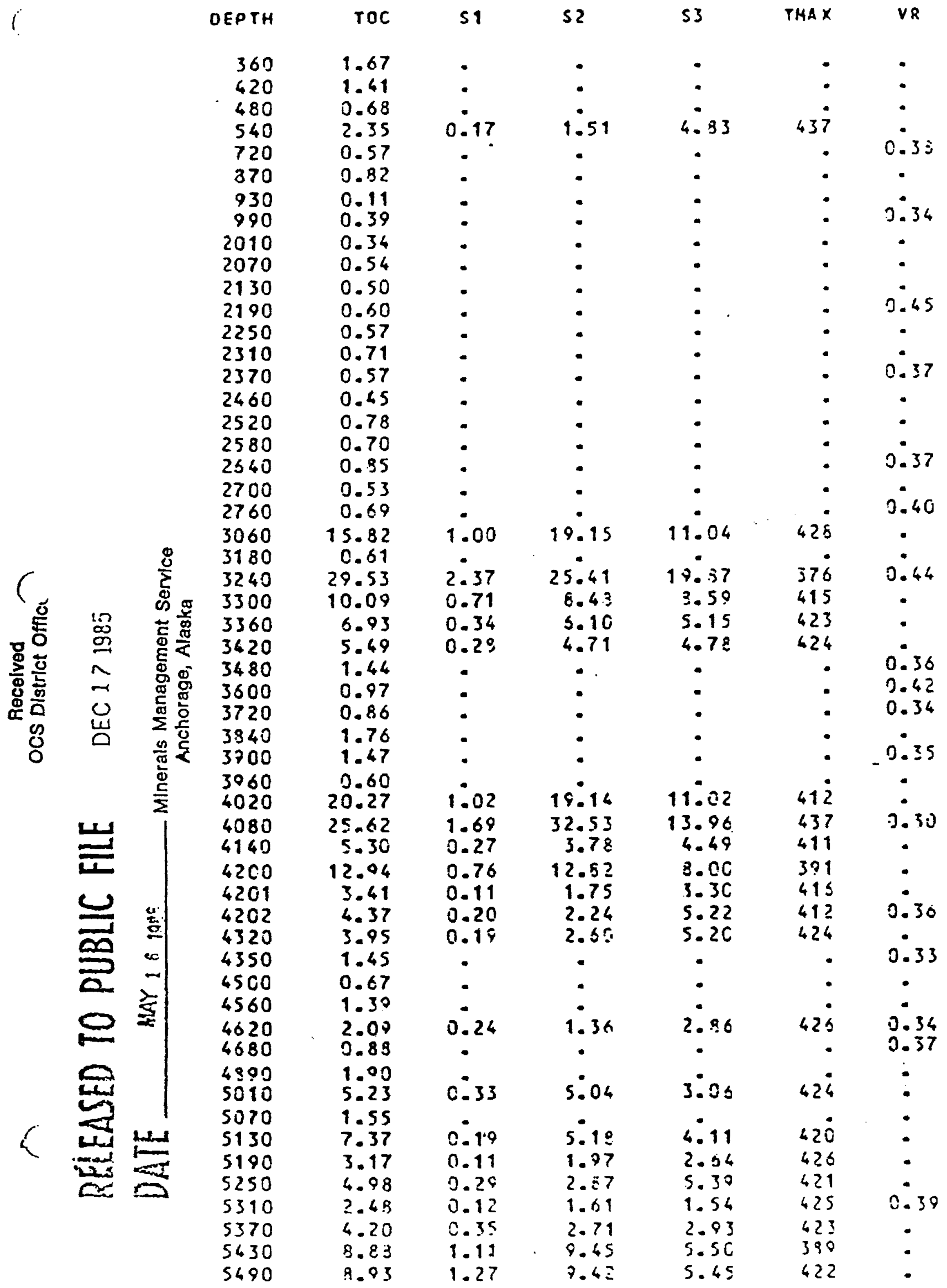




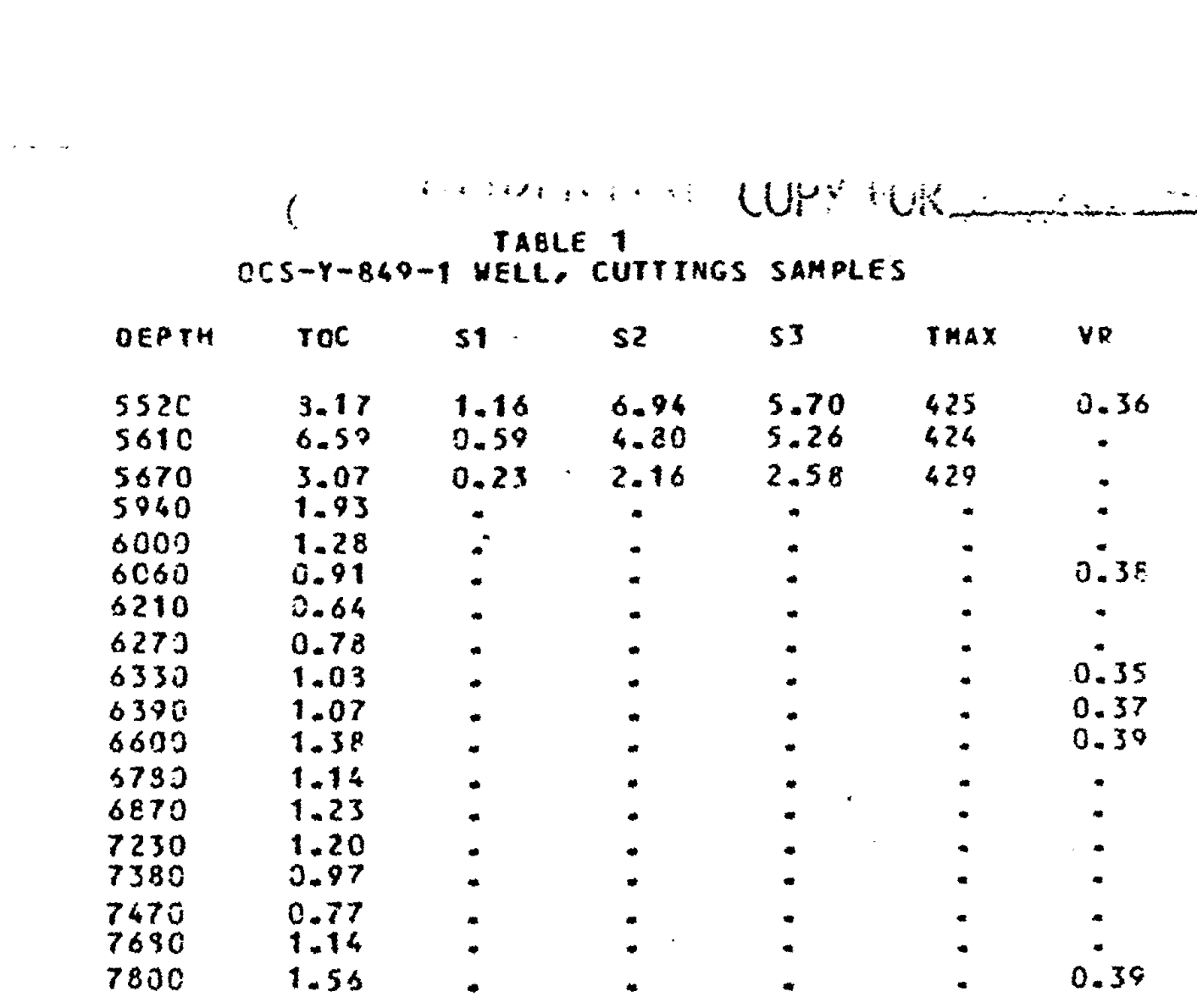


i U. TABLE 2 OCS-Y-949-1 WELL, SIDEYALL CORES

\begin{tabular}{|c|c|c|c|c|c|c|}
\hline DEPTH & $T O C$ & 51 & 52 & 53 & $\operatorname{Trax}$ & YR \\
\hline $\begin{array}{l}3231 \\
3334 \\
3519 \\
3666\end{array}$ & $\begin{array}{l}1.53 \\
0.95 \\
1.25 \\
1.79\end{array}$ & $\begin{array}{l}3.10 \\
0.05 \\
0.13 \\
0.11\end{array}$ & $\begin{array}{l}0.76 \\
0.45 \\
0.81 \\
1.27\end{array}$ & $\begin{array}{l}4.94 \\
8.97 \\
1.14 \\
8.16\end{array}$ & $\begin{array}{l}426 \\
430 \\
425^{\circ} \\
427\end{array}$ & $\begin{array}{l}0.25 \\
0.35 \\
0.27 \\
0.25\end{array}$ \\
\hline $\begin{array}{l}3732 \\
3780 \\
3870 \\
3902\end{array}$ & $\begin{array}{l}1.70 \\
1.64 \\
2.31 \\
1.65\end{array}$ & $\begin{array}{l}0.15 \\
0.11 \\
0.11 \\
0.09\end{array}$ & $\begin{array}{l}1.25 \\
1.11 \\
1.13 \\
0.87\end{array}$ & $\begin{array}{l}8.28 \\
5.42 \\
7.52 \\
5.70\end{array}$ & $\begin{array}{l}420 \\
428 \\
423 \\
424\end{array}$ & $\begin{array}{l}0.28 \\
0.34 \\
0.29 \\
0.25\end{array}$ \\
\hline $\begin{array}{l}3978 \\
4120\end{array}$ & $\begin{array}{l}1.36 \\
1.51\end{array}$ & $\begin{array}{l}0.05 \\
0.10\end{array}$ & $\begin{array}{l}0.69 \\
0.37\end{array}$ & $\begin{array}{l}4.46 \\
6.09\end{array}$ & $\begin{array}{l}426 \\
425\end{array}$ & $\begin{array}{l}0.25 \\
0.24\end{array}$ \\
\hline $\begin{array}{l}4243 \\
4426\end{array}$ & $\begin{array}{l}1.14 \\
1.77\end{array}$ & $\begin{array}{l}0.02 \\
0.10\end{array}$ & $\begin{array}{l}0.37 \\
1.43\end{array}$ & $\begin{array}{r}5.55 \\
11.88\end{array}$ & $\begin{array}{l}425 \\
426\end{array}$ & $\begin{array}{l}0.39 \\
0.29\end{array}$ \\
\hline $\begin{array}{l}4445 \\
4495\end{array}$ & $\begin{array}{l}2.70 \\
1.52\end{array}$ & $\begin{array}{l}3.13 \\
0.05\end{array}$ & $\begin{array}{l}2.07 \\
0.63\end{array}$ & $\begin{array}{l}8.34 \\
5.97\end{array}$ & $\begin{array}{l}427 \\
424\end{array}$ & $\begin{array}{l}0.29 \\
0.31\end{array}$ \\
\hline $\begin{array}{l}4576 \\
4520\end{array}$ & $\begin{array}{l}1.85 \\
2.24\end{array}$ & $\begin{array}{l}0.08 \\
0.10\end{array}$ & $\begin{array}{l}0.89 \\
1.50\end{array}$ & $\begin{array}{r}5.36 \\
10.95\end{array}$ & $\begin{array}{l}425 \\
425\end{array}$ & $\begin{array}{l}0.30 \\
0.31\end{array}$ \\
\hline $\begin{array}{l}4583 \\
4764 \\
4944 \\
4910 \\
4970 \\
5004 \\
5063 \\
5100 \\
5290\end{array}$ & $\begin{array}{l}1.45 \\
1.58 \\
1.24 \\
1.05 \\
1.10 \\
1.55 \\
1.34 \\
2.03 \\
0.91\end{array}$ & $\begin{array}{l}0.03 \\
0.07 \\
0.06 \\
0.07 \\
0.06 \\
0.08 \\
0.07 \\
0.08 \\
0.04\end{array}$ & $\begin{array}{l}0.40 \\
0.80 \\
0.60 \\
0.80 \\
0.43 \\
1.11 \\
0.76 \\
1.33 \\
0.42\end{array}$ & $\begin{array}{r}11.53 \\
6.72 \\
1.58 \\
7.05 \\
4.29 \\
5.47 \\
5.61 \\
3.93 \\
2.97\end{array}$ & $\begin{array}{l}426 \\
425 \\
428 \\
427 \\
428 \\
427 \\
427 \\
426 \\
432\end{array}$ & $\begin{array}{l}0.33 \\
0.30 \\
0.32 \\
0.36 \\
0.25 \\
0.26 \\
0.30 \\
0.24 \\
0.37\end{array}$ \\
\hline $\begin{array}{l}5335 \\
54 C 1\end{array}$ & $\begin{array}{l}0.30 \\
1.54\end{array}$ & $\begin{array}{l}0.09 \\
0.13\end{array}$ & $\begin{array}{l}0.56 \\
1.14\end{array}$ & $\begin{array}{r}4.68 \\
11.41\end{array}$ & $\begin{array}{l}426 \\
428\end{array}$ & $\begin{array}{l}0.38 \\
0.39\end{array}$ \\
\hline $\begin{array}{l}5562 \\
5765\end{array}$ & $\begin{array}{l}1.25 \\
0.94\end{array}$ & $\begin{array}{l}0.04 \\
0.03\end{array}$ & $\begin{array}{l}0.71 \\
0.35\end{array}$ & $\begin{array}{l}6.67 \\
1.21\end{array}$ & $\begin{array}{l}425 \\
426\end{array}$ & $\begin{array}{l}0.38 \\
0.33\end{array}$ \\
\hline $\begin{array}{l}5003 \\
6072\end{array}$ & $\begin{array}{l}3.89 \\
1.29\end{array}$ & $\begin{array}{l}0.24 \\
0.04\end{array}$ & $\begin{array}{l}3.05 \\
0.57\end{array}$ & $\begin{array}{l}5.54 \\
5.11\end{array}$ & $\begin{array}{l}424 \\
432\end{array}$ & $\begin{array}{l}0.37 \\
0.33\end{array}$ \\
\hline $\begin{array}{l}6074 \\
0212\end{array}$ & $\begin{array}{r}1.50 \\
10.05\end{array}$ & $\begin{array}{l}0.14 \\
0.24\end{array}$ & $\begin{array}{l}0.96 \\
4.38\end{array}$ & $\begin{array}{r}8.27 \\
10.39\end{array}$ & $\begin{array}{l}425 \\
419\end{array}$ & $\begin{array}{l}0.41 \\
0.35\end{array}$ \\
\hline $\begin{array}{l}6380 \\
6500 \\
5600 \\
6700 \\
0905 \\
7000 \\
7200 \\
7300 \\
7450 \\
7653 \\
7750\end{array}$ & $\begin{array}{l}1.29 \\
0.74 \\
2.63 \\
2.02 \\
0.75 \\
1.34 \\
1.09 \\
1.09 \\
1.05 \\
0.95 \\
1.04\end{array}$ & $\begin{array}{l}0.04 \\
0.03 \\
0.07 \\
0.04 \\
0.07 \\
0.25 \\
0.13 \\
0.07 \\
0.03 \\
0.03 \\
3.07\end{array}$ & $\begin{array}{l}0.68 \\
0.15 \\
1.53 \\
0.49 \\
0.57 \\
1.40 \\
1.23 \\
0.92 \\
0.60 \\
0.77 \\
0.93\end{array}$ & $\begin{array}{l}4.08 \\
1.03 \\
5.11 \\
1.42 \\
3.38 \\
2.56 \\
5.46 \\
5.95 \\
6.55 \\
2.72 \\
4.01\end{array}$ & $\begin{array}{l}429 \\
421 \\
427 \\
422 \\
428 \\
427 \\
427 \\
425 \\
430 \\
430 \\
425\end{array}$ & $\begin{array}{l}0.38 \\
0.40 \\
0.37 \\
0.37 \\
0.36 \\
0.38 \\
0.42 \\
0.40 \\
0.43 \\
0.40 \\
0.44\end{array}$ \\
\hline
\end{tabular}

Recelved

OCS District Office

\section{DARE MAY 161988 \\ MAY 161988 \\ REIEASED TO PUBLIC FILE}

